\title{
A Study of Weighted Polynomial Approximations with Several Variables (I)
}

\author{
Ryozi Sakai \\ Department of Mathematics, Meijo University, Nagoya, Japan \\ Email: ryozi@crest.ocn.ne.jp
}

How to cite this paper: Sakai, R. (2017) A Study of Weighted Polynomial Approximations with Several Variables (I). Applied Mathematics, 8, 1267-1306.

https://doi.org/10.4236/am.2017.89095

Received: August 3, 2017

Accepted: September 16, 2017

Published: September 19, 2017

Copyright $\odot 2017$ by author and Scientific Research Publishing Inc. This work is licensed under the Creative Commons Attribution International License (CC BY 4.0).

http://creativecommons.org/licenses/by/4.0/

\begin{abstract}
In this paper, we investigate the weighted polynomial approximations with several variables. Our study relates to the approximation for $W f \in L^{p}\left(\mathbb{R}^{s}\right)$ by weighted polynomials. Then we will estimate the degree of approximation.

\section{Keywords}

Weighted Polynomial Approximations with Several Variables, the Degree of Approximations
\end{abstract}

\section{Introduction}

Let $\mathbb{R}^{s}:=\mathbb{R} \times \mathbb{R} \times \cdots \times \mathbb{R}$ ( $s$ times, $s \geq 1$ integer) be the direct product space, and let $W\left(x_{1}, x_{2}, \cdots, x_{s}\right):=w_{1}\left(x_{1}\right) w_{2}\left(x_{2}\right) \cdots w_{s}\left(x_{s}\right)$, where $w_{i}\left(x_{i}\right) \geq 0$ are even weight functions. We suppose that for every nonnegative integer $n$,

$$
\int_{0}^{\infty} x^{n} w_{i}(x) \mathrm{d} x<\infty, n=0,1,2, \cdots, i=1,2, \cdots, s .
$$

In this paper, we will study to approximate the real-valued weighted function $(W f)\left(x_{1}, x_{2}, \cdots, x_{s}\right)$ by weighted polynomials $(W P)\left(x_{1}, x_{2}, \cdots, x_{s}\right)$, where $P\left(x_{1}, x_{2}, \cdots, x_{s}\right) \in \mathcal{P}_{n, n, \cdots, n}\left(\mathbb{R}^{s}\right)$. Here, $\mathcal{P}_{n, n, \cdots, n}\left(\mathbb{R}^{s}\right)\left(=: \mathcal{P}_{n ; s}\left(\mathbb{R}^{s}\right)\right)$ means a class of all polynomials with at most $n$-degree for each variable $x_{i}, i=1,2, \cdots, s$. We need to define the norms. Let $0<p \leq \infty$, and let $f: \mathbb{R}^{s} \rightarrow \mathbb{R}$ be measurable. Then we define

$$
\|W f\|_{L^{p}\left(\mathbb{R}^{s}\right)}:=\left\{\begin{array}{lc}
{\left[\int_{-\infty}^{\infty} \cdots \int_{-\infty}^{\infty}\left|(W f)\left(x_{1}, \cdots, x_{s}\right)\right|^{p} \mathrm{~d} x_{1} \cdots \mathrm{d} x_{s}\right]^{1 / p},} & \text { if } 0<p<\infty ; \\
\sup _{\left(x_{1}, \cdots, x_{s}\right) \in \mathbb{R}^{s}}\left|(W f)\left(x_{1}, \cdots, x_{s}\right)\right|, & \text { if } p=\infty .
\end{array}\right.
$$


We assume that for $0<p \leq \infty$ the integral is independent of the order of integration with respect to each $x_{i}, i=1,2, \cdots, s$. When $\|W f\|_{L^{p}\left(\mathbb{R}^{s}\right)}<\infty$, we write $W f \in L^{p}\left(\mathbb{R}^{s}\right)$. If $p=\infty$, we require that $f$ is continuous and $\lim _{|X| \rightarrow \infty}(W f)(X)=0$, where $|X|=\left|\left(x_{1}, \cdots, x_{s}\right)\right|=\max \left|x_{i}\right| ; i=1,2, \cdots, s$.

Our purpose in this paper is to approximate the weighted function $W f \in L^{p}\left(\mathbb{R}^{s}\right)$ by weighted polynomials $W P ; P \in \mathcal{P}_{n ; s}\left(\mathbb{R}^{s}\right)$. The paper is arranged as the following. In Section 2, we give the definition of the weights which are treated in this paper. In Section 3, we consider the approximation for the functions in $L^{p}\left(\mathbb{R}^{s}\right)$. In Section 4 , we consider a property of higher order derivatives. In Section 5, we estimate the degree of approximations. In Section 6, we consider the approximation for the functions with bounded variation. In Section 7, we consider the approximation of the Lipschitz-type functions. In Section 8 , we treat the functions with higher order derivatives.

\section{Class of Weight Functions and Preliminaries}

Throughout the paper $C, C_{1}, C_{2}, \cdots$ denote positive constants independent of $n, x, t$ or polynomials $P(x)$. The same symbol does not necessarily denote the same constant in different occurrences. Let $f(x) \sim g(x)$ mean that there exists a constant $C>0$ such that $C^{-1} f(x) \leq g(x) \leq C f(x)$ holds for all $x \in I$, where $I \subset \mathbb{R}$ is a subset.

We say that $f: \mathbb{R} \rightarrow[0, \infty)$ is quasi-increasing if there exists $C>0$ such that $f(x) \leq C f(y)$ for $0<x<y$. Hereafter we consider following weights.

Definition 2.1. Let $Q: \mathbb{R} \rightarrow[0, \infty)$ be a continuous and even function, and satisfy the following properties:

(a) $Q^{\prime}(x)$ is continuous in $\mathbb{R}$, with $Q(0)=0$.

(b) $Q^{\prime \prime}(x)$ exists and is positive in $\mathbb{R} \backslash\{0\}$.

(c) $\lim _{x \rightarrow \infty} Q(x)=\infty$

(d) The function

$$
T(x):=\frac{x Q^{\prime}(x)}{Q(x)}, x \neq 0
$$

is quasi-increasing in $(0, \infty)$, with

$$
T(x) \geq \Lambda>1, x \in \mathbb{R} \backslash\{0\} .
$$

(e) There exists $C_{1}>0$ such that

$$
\frac{Q^{\prime \prime}(x)}{\left|Q^{\prime}(x)\right|} \leq C_{1} \frac{\left|Q^{\prime}(x)\right|}{Q(x)} \text {, a.e. } x \in \mathbb{R} \text {. }
$$

Then we write $w=\exp (-Q) \in \mathcal{F}\left(C^{2}\right)$.

Moreover, if there also exists a compact subinterval $J(\ni 0)$ of $\mathbb{R}$, and $C_{2}>0$ such that

$$
\frac{Q^{\prime \prime}(x)}{\left|Q^{\prime}(x)\right|} \geq C_{2} \frac{\left|Q^{\prime}(x)\right|}{Q(x)} \text {, a.e. } x \in \mathbb{R} \backslash J,
$$


then we write $w=\exp (-Q) \in \mathcal{F}\left(C^{2}+\right)$. If $T(x)$ is bounded, then the weight $w=\exp (-Q) \in \mathcal{F}\left(C^{2}+\right)$ is called a Freud-type weight, and if $T(x)$ is unbounded, then $w$ is called an Erdös-type weight.

For $w(x)=\exp (-Q(x)) \in \mathcal{F}\left(C^{2}+\right), Q \in C^{3}(\mathbb{R} \backslash\{0\})$, if there exists $K>0$ such that for $|x| \geq K$,

$$
\left|\frac{Q^{\prime \prime \prime}(x)}{Q^{\prime \prime}(x)}\right| \leq C\left|\frac{Q^{\prime \prime}(x)}{Q^{\prime}(x)}\right|,
$$

and there exist $\lambda, C>0$ such that for $0<\lambda<\frac{3}{2}$,

$$
\frac{\left|Q^{\prime}(x)\right|}{Q(x)^{\lambda}} \leq C,
$$

then we write $w \in \mathcal{F}_{\lambda}\left(C^{3}+\right)$. Furthermore, if

$$
\left|\frac{Q^{(4)}(x)}{Q^{(3)}(x)}\right| \leq C\left|\frac{Q^{\prime \prime \prime}(x)}{Q^{\prime \prime}(x)}\right| \sim\left|\frac{Q^{\prime \prime}(x)}{Q^{\prime}(x)}\right|
$$

and the inequality (2.2) with $0<\lambda<\frac{4}{3}$ hold, then we write $w \in \mathcal{F}_{\lambda}\left(C^{4}+\right)$.

We have some examples satisfying Definition 2.1.

Example 2.2 (cf. [1] [2]). (1) If an exponential $Q(x)$ satisfies

$$
1<\Lambda_{1} \leq \frac{\left(x Q^{\prime}(x)\right)^{\prime}}{Q^{\prime}(x)} \leq \Lambda_{2},
$$

where $\Lambda_{i}, i=1,2$ are constants, then we call $w=\exp (-Q(x))$ the Freud weight. The class $\mathcal{F}\left(C^{2}+\right)$ contains the Freud weights.

(2) For $\alpha>1, l \geq 1$ we define

$$
Q(x)=Q_{l ; \alpha}(x)=\exp _{l}\left(|x|^{\alpha}\right)-\exp _{l}(0),
$$

where $\exp _{l}(x)=\exp (\exp (\exp \cdots \exp x) \cdots)(l$ times $)$. Moreover, we define

$$
Q_{l ; \alpha, m}(x)=|x|^{m}\left\{\exp _{l}\left(|x|^{\alpha}\right)-\alpha^{*} \exp _{l}(0)\right\}, \alpha+m>1, m \geq 0, \alpha \geq 0,
$$

where $\alpha^{*}=0$ if $\alpha=0$, and otherwise $\alpha^{*}=1$. We note that $Q_{1 ; 0, m}$ gives a Freud-type weight, that is, $T(x)$ is bounded..

(3) We define

$$
Q_{\alpha}(x)=(1+|x|)^{|x|^{\alpha}}-1, \alpha>1 .
$$

(4) Let $w=\exp (-Q) \in \mathcal{F}\left(C^{2}+\right)$, and let us define

$$
\mu_{+}:=\limsup _{x \rightarrow \infty} \frac{Q^{\prime \prime}(x)}{Q^{\prime}(x)} / \frac{Q^{\prime}(x)}{Q(x)}, \mu_{-}:=\liminf _{x \rightarrow \infty} \frac{Q^{\prime \prime}(x)}{Q^{\prime}(x)} / \frac{Q^{\prime}(x)}{Q(x)} \text {. }
$$

If $\mu_{+}=\mu_{-}$, then we say that the weight $w$ is regular. All weights in examples (1), (2) and (3) are regular. 
(5) More generally we can give the examples of weights $w \in \mathcal{F}_{\lambda}\left(C^{3}+\right)$. If the weight $w$ is regular and if $Q \in \mathbf{C}^{3}(\mathbb{R} \backslash\{0\})$ satisfies (2.1), then for the regular weights we have $w \in \mathcal{F}_{\lambda}\left(C^{3}+\right)$ (see [3], Corollary $5.5(5.8)$ ).

The following fact is very important for our study.

Proposition 2.3 ([3], Theorem 4.1 and (4.11)). Let $0<\lambda<3 / 2$ and $\alpha \in \mathbb{R}$. Then for $w=\exp (-Q) \in \mathcal{F}_{\lambda}\left(C^{3}+\right)$, we can construct a new weight $w_{\alpha} \in \mathcal{F}\left(C^{2}+\right)$ such that

$$
T_{w}(x)^{\alpha} w(x) \sim w_{\alpha}(x) \text { on } \mathbb{R},
$$

and for some $C \geq 1$,

$$
a_{n / C}\left(w_{\alpha}\right) \leq a_{n}(w) \leq a_{C n}\left(w_{\alpha}\right) \text { and } T_{w_{\alpha}}(x) \sim T_{w}(x)=T(x),
$$

where $a_{n}\left(w_{\alpha}\right)$ and $a_{n}(w)$ are MRS-numbers for the weight $w_{\alpha}$ and $w$, respectively, and $T_{w_{\alpha}}$ and $T_{w}$ are correspond for $w_{\alpha}$ or $w$, respectively.

Let $\left\{p_{n}\right\}$ be orthonormal polynomials with respect to a weight $w$, that is, $p_{n}$ is the polynomial of degree $n$ such that

$$
\int_{-\infty}^{\infty} p_{n}(x) p_{m}(x) w^{2}(x) \mathrm{d} x=\delta_{m n} \text { (the Kronecker delta). }
$$

For $1 \leq p \leq \infty$, we denote by $L^{p}(\mathbb{R})$ the usual $L^{p}$ space on $\mathbb{R}$ (here for $p=\infty$, if $w f \in L^{\infty}(\mathbb{R})$, then we require $f$ to be continuous, and $w f$ to have limit 0 at $\pm \infty)$. For $w f \in L^{p}(\mathbb{R})$, we set

$$
\begin{aligned}
& s_{n}(f, x):=\sum_{k=0}^{n-1} b_{k}(f) p_{k}(x), \\
& \text { where } b_{k}(f)=\int_{-\infty}^{\infty} f(t) p_{k}(t) w^{2}(t) \mathrm{d} t
\end{aligned}
$$

for $n \in \mathbb{N}$ (the partial sum of Fourier-type series). The de la Vallée Poussin mean of order $n$ is defined by

$$
v_{n}(f, x):=\sum_{j=n+1}^{2 n} s_{j}(f, x) .
$$

Let $w \in \mathcal{F}\left(C^{2}+\right)$. We need the Mhaskar-Rakhmanov-Saff numbers (MRS-numbers) $a_{x}$;

$$
x=\frac{2}{\pi} \int_{0}^{1} \frac{a_{x} u Q^{\prime}\left(a_{x} u\right)}{\left(1-u^{2}\right)^{1 / 2}} \mathrm{~d} u, x>0
$$

We easily see

$$
\lim _{x \rightarrow \infty} a_{x}=\infty \text { and } \lim _{x \rightarrow+0} a_{x}=0
$$

and

$$
\lim _{x \rightarrow \infty} \frac{a_{x}}{x}=0 \text { and } \lim _{x \rightarrow+0} \frac{a_{x}}{x}=\infty .
$$

For $w f \in L_{p}(\mathbb{R})(1 \leq p \leq \infty)$, the degree of weighted polynomial approximation 
is defined by

$$
E_{n, p}(w ; f):=\inf _{P \in \mathcal{P}_{n}}\|w(f-P)\|_{L^{p}(\mathbb{R})} .
$$

\section{Approximations for $L_{p}$-Functions}

In this section, we treat the function such as $W f \in L^{p}\left(\mathbb{R}^{s}\right)$, where $\left.1 \leq p \leq \infty\right)$, and if $p=\infty$, then we suppose that $W f$ is continuous and $\lim _{|X| \rightarrow \infty}(W f)(X)=0$. For any multivariate point $X=\left(x_{1}, \cdots, x_{s}\right) \in \mathbb{R}^{s}$, we consider the weights;

$$
W(X):=\prod_{j=1}^{s} w_{j}\left(x_{j}\right)=\prod_{j=1}^{s} \exp \left(-Q_{j}\left(x_{j}\right)\right) .
$$

As shown under, we will also use $X(u):=\left(u_{1}, u_{2}, \cdots, u_{s}\right)$. Let $w_{i}=\exp \left(-Q_{i}\right) \in \mathcal{F}_{\lambda}\left(C^{3}+\right), \quad 0<\lambda<3 / 2, \quad i=1,2, \cdots, s$. From Proposition 2.3 we see $T_{i}^{1 / 4} w_{i} \sim w_{i, 1 / 4} \in \mathcal{F}\left(C^{2}+\right), i=1,2, \cdots, s$. Then we admit to write $T_{i}^{1 / 4} w_{i} \in \mathcal{F}\left(C^{2}+\right)$. For the weight $W$ we construct the modulus of continuity of $f$. It involves the function

$$
\Phi_{t, i}\left(x_{i}\right):=\sqrt{1-\frac{\left|x_{i}\right|}{\sigma_{i}(t)}}+\frac{1}{\sqrt{T_{i}\left(\sigma_{i}(t)\right)}}, i=1,2, \cdots, s,
$$

where $\sigma_{i}(t)$ is defined by

$$
\sigma_{i}(t):=\inf \left\{a_{n}^{(i)}: \frac{a_{n}^{(i)}}{n} \leq t\right\}, t>0,
$$

where $a_{n}^{(i)}$ is the MRS-number for the weight $w_{i}(x)$. If $a_{n}^{(i)} / n=t$, then we have $\sigma_{i}(t)=a_{n}^{(i)}$. In the sequel, if $1 \leq j \leq s$ is an integer, then $\|f\|_{p ; j}$ will denote the $L^{p}$ norm of $f$ taken with respect to the $j$-th variable. This is a function of the remaining $(s-1)$ variables. For each fixed $\hat{X}_{j}:=\left(x_{1}, \cdots, x_{j-1}, x_{j+1}, \cdots, x_{s}\right) \in \mathbb{R}_{j}^{s-1}$, we write

$$
f_{\hat{x}_{j}}(x):=f\left(x_{1}, \cdots, x_{j-1}, x, x_{j+1}, \cdots, x_{s}\right), j=1,2, \cdots, s .
$$

Using

$$
\Delta_{h} f_{\hat{X}_{j}}(x):=f_{\hat{X}_{j}}\left(x+\frac{h}{2}\right)-f_{\hat{X}_{j}}\left(x-\frac{h}{2}\right)
$$

we define the modulus of continuity. For the Freud-type weight, we define

$$
\begin{aligned}
& \bar{\omega}_{p, j}\left(f_{\hat{X}_{j}}, w_{j} ; t\right) \\
& :=\left(\frac{1}{t} \int_{0}^{t}\left\|w_{j}(x)\left(\Delta_{h} f_{\hat{X}_{j}}(x)\right)\right\|_{L^{p}\left(|x| \leq \sigma_{j}(2 t)\right)}^{p} \mathrm{~d} h\right)^{1 / p} \\
& \quad+\inf _{c_{j}(\text { constant })}\left\|\left(f_{\hat{X}_{j}}(x)-c_{j}\right) w_{j}(x)\right\|_{L^{p}\left(|x| \leq \sigma_{j}(4 t)\right)} .
\end{aligned}
$$

If $w_{j}$ is Erdös-type, then we define 


$$
\begin{aligned}
& \bar{\omega}_{p, j}\left(f_{\hat{X}_{j}}, w_{j} ; t\right) \\
& :=\left(\frac{1}{t} \int_{0}^{t}\left\|w_{j}(x)\left(\Delta_{h \Phi_{t, j}(x)} f_{\hat{X}_{j}}(x)\right)\right\|_{L^{p}\left(|x| \leq \sigma_{j}(2 t)\right)}^{p} \mathrm{~d} h\right)^{1 / p} \\
& \quad+\inf _{\left.c_{j} \text { (constant) }\right)}\left\|\left(f_{\hat{X}_{j}}(x)-c_{j}\right) w_{j}(x)\right\|_{L^{p}\left(|x| \leq \sigma_{j}(4 t)\right)} .
\end{aligned}
$$

We remark that if $T_{j}(x)$ is bounded, then we see $\Phi_{t, j}(x) \sim 1$, so we do not need the definition for the Freud-type weight.

Let $v_{n}$ be the de la Vallée Poussin mean opetator, and let $v_{n, j}(f), j=1,2, \cdots, s$ denote the operation to $f$ with respect to $j$-th co-ordinate, and $v_{n}^{[j]}$ will denote the operator $v_{n}$ applied to $f$ with respect to each of the first $j$ co-ordinates. Clearly,

$$
v_{n}^{[1]}(f)=v_{n, 1}(f), v_{n}^{[j]}(f)=v_{n}^{[j-1]}\left(v_{n, j}(f)\right), j=2,3, \cdots, s .
$$

Let $a_{n}^{(j)}$ be the MRS-number for the weight $w_{j}=\exp \left(-Q_{j}\right)$.

First, we consider the following Proposition.

Proposition 3.1 ([4], Theorem 3.14). For $1 \leq p<\infty, C_{c}\left(\mathbb{R}^{s}\right)$ is dence in $L^{p}\left(\mathbb{R}^{s}\right)$, where $C_{c}\left(\mathbb{R}^{s}\right)$ is a set of all continuous functions with a compact support on $\mathbb{R}^{s}$.

From this proposition, for any $\varepsilon>0$ there exist a constant $K>0$ and a continuous function $f_{K}$ with a compact support $[-K, K]^{s}$ such that

$$
\left\|W(X)\left(f(X)-f_{K}(X)\right)\right\|_{L^{p}(|X| \leq K)}<\varepsilon .
$$

Then we give the following assumption:

Assumption 3.2. In (3.3) we suppose that for every co-ordinate $x_{j}, j=1,2, \cdots, s$

$$
\left\|w_{j}(x)\left(f_{\hat{X}_{j}}(x)-\left(f_{K}\right)_{\hat{X}_{j}}(x)\right)\right\|_{L^{p}(|x| \leq K)}<\varepsilon
$$

holds.

We define a new class of functions $L_{p}^{*}\left(\mathbb{R}^{s}\right), 1 \leq p \leq \infty$ as follows:

$$
L_{W}^{p^{*}}\left(\mathbb{R}^{s}\right):=\left\{f \mid W f \in L_{p}\left(\mathbb{R}^{s}\right) \operatorname{holds}(3.4)\right\},
$$

where if $p=\infty$, then $L_{w}^{p^{*}}\left(\mathbb{R}^{s}\right)=L_{w}^{p}\left(\mathbb{R}^{s}\right)$ and we suppose that $f$ is continuous and

$$
\lim _{|X| \rightarrow \infty} W(X) f(X)=0
$$

(we write this fact as $W f \in C_{0}\left(\mathbb{R}^{s}\right)$ ). We state the theorem in this section.

Theorem 3.3. (1) We suppose

$$
\begin{gathered}
w_{j}=\exp \left(-Q_{j}\right) \in \mathcal{F}_{\lambda}\left(C^{3}+\right)(0<\lambda<3 / 2), j=1,2, \cdots, s, \text { and let } \\
T_{j}\left(a_{n}^{(j)}\right) \leq c\left(\frac{n}{a_{n}^{(j)}}\right)^{2 / 3}, j=1,2, \cdots, s .
\end{gathered}
$$

Let $n \geq 1,1 \leq p \leq \infty$. Then we have 


$$
\begin{aligned}
& \left\|W\left(f-v_{n}^{[s]}(f)\right)\right\|_{L^{p}\left(\mathbb{R}^{s}\right)} \\
& \leq \sum_{j=1}^{s} C_{j}\left\|\left(\prod_{1 \leq i \leq s, i \neq j} w_{i}\right)\left(\prod_{k=1}^{j-1} T_{k}^{1 / 4}\right) \bar{\omega}_{p, j}\left(f_{\hat{X}_{j}}, T_{j}^{1 / 4} w_{j} ; c_{j} \frac{a_{n}^{(j)}}{n}\right)\right\|_{L^{p}\left(\mathbb{R}_{j}^{s-1}\right)},
\end{aligned}
$$

where

$$
\mathbb{R}_{j}^{s-1}:=\left\{\left(x_{1}, \cdots, x_{j-1}, x_{j+1}, \cdots, x_{s}\right)\right\},
$$

and $\prod_{k=1}^{0} T_{k}^{1 / 4}=1$. Especially $f \in L_{T^{(s)} W}^{p^{*}}\left(\mathbb{R}^{s}\right)$, then we have

$$
\sum_{j=1}^{s} C_{j}\left\|\left(\prod_{1 \leq i \leq s, i \neq j} w_{i}\right)\left(\prod_{k=1}^{j-1} T_{k}^{1 / 4}\right) \bar{\omega}_{p, j}\left(f_{\hat{X}_{j}}, T_{j}^{1 / 4} w_{j} ; c_{j} \frac{a_{n}^{(j)}}{n}\right)\right\|_{L^{p}\left(\mathbb{R}_{j}^{s-1}\right)} \rightarrow 0
$$

as $n \rightarrow \infty$.

(2) We suppose $w_{j}=\exp \left(-Q_{j}\right) \in \mathcal{F}\left(C^{2}+\right), j=1,2, \cdots, s$, and let (3.6) holds. Let $n \geq 1,1 \leq p \leq \infty$. Then we have

$$
\left\|\frac{W\left(f-v_{n}^{[s]}(f)\right)}{\prod_{k=1}^{s} T_{k}^{1 / 4}}\right\|_{L^{p}\left(\mathbb{R}^{s}\right)} \leq \sum_{j=1}^{s} C_{j}\left\|\left(\prod_{1 \leq i \leq s, i \neq j} w_{i}\right) \bar{\omega}_{p, j}\left(f_{\hat{X}_{j}}, w_{j} ; c_{j} \frac{a_{n}^{(j)}}{n}\right)\right\|_{L^{p}\left(\mathbb{R}_{j}^{s-1}\right)} .
$$

Especially $f \in L_{w}^{p^{*}}\left(\mathbb{R}^{s}\right)$, then we have

$$
\sum_{j=1}^{s} C_{j}\left\|\left(\prod_{1 \leq i \leq s, i \neq j} w_{i}\right) \bar{\omega}_{p, j}\left(f_{\hat{X}_{j}}, w_{j} ; c_{j} \frac{a_{n}^{(j)}}{n}\right)\right\|_{L^{p}\left(\mathbb{R}_{j}^{s-1}\right)} \rightarrow 0 \text { as } n \rightarrow \infty .
$$

First we will show (3.7). We need some preliminaries.

Proposition 3.4 ([5], Theorem 1). Let $1 \leq p \leq \infty$.

(1) We assume that $w \in \mathcal{F}\left(C^{2}+\right)$ satisfies $T\left(a_{n}\right) \leq C\left(n / a_{n}\right)^{2 / 3}$. Then there exists a constant $C>0$ such that when $w g \in L_{p}(\mathbb{R})$, then

$$
\left\|\frac{w v_{n}(g)}{T^{1 / 4}}\right\|_{L^{p}(\mathbb{R})} \leq C\|w g\|_{L^{p}(\mathbb{R})},
$$

and so,

$$
\left\|w v_{n}(g)\right\|_{L^{p}(\mathbb{R})} \leq C T\left(a_{n}\right)^{1 / 4}\|w g\|_{L^{p}(\mathbb{R})} .
$$

(2) We assume that $w \in \mathcal{F}_{\lambda}\left(C^{3}+\right)(0<\lambda<3 / 2)$ satisfies $T\left(a_{n}\right) \leq C\left(n / a_{n}\right)^{2 / 3}$. Then there exists a constant $C>0$ such that if $T^{1 / 4} w g \in L^{p}(\mathbb{R})$, then

$$
\left\|w v_{n}(g)\right\|_{L^{p}(\mathbb{R})} \leq C\left\|T^{1 / 4} w g\right\|_{L^{p}(\mathbb{R})} .
$$

Proposition 3.5 ([5], Corollary 6.2 (6.5)). Let $1 \leq p \leq \infty$.

(1) Let $w \in \mathcal{F}\left(C^{2}+\right)$, and $n \geq 1$ be an integer. Then

$$
\left\|\frac{w\left(g-v_{n}(g)\right)}{T^{1 / 4}}\right\|_{L^{p}(\mathbb{R})} \leq C E_{n, p}(w ; g),
$$


where $C$ do not depend on $g$ and $n$.

(2) Let $w \in \mathcal{F}_{\lambda}\left(C^{3}+\right)(0<\lambda<3 / 2)$, and $n \geq 1$ be an integer. Then

$$
\left\|w\left(g-v_{n}(g)\right)\right\|_{L^{p}(\mathbb{R})} \leq C E_{n, p}\left(T^{1 / 4} w ; g\right),
$$

where $C$ do not depend on $g$ and $n$.

Proposition 3.6. ([6]) Let $w=\exp (-Q) \in \mathcal{F}\left(C^{2}+\right)$, and let $0<p \leq \infty$. Then there exist $n_{0} \in \mathbb{N}$ and positive constants $C_{1}, C_{2}$ such that for every $w g \in L^{p}(\mathbb{R})$ (and for $p=\infty$, we require $g$ to be continuous, and $w f$ to vanish at $\pm \infty$ ) and every $n \geq n_{0}$,

$$
E_{n, p}(g ; w) \leq C_{1} \bar{\omega}_{p}\left(g, w ; C_{2} \frac{a_{n}}{n}\right),
$$

where $n_{0}$ and $C_{1}, C_{2}$ do not depend on $g$ and $n$, and $\omega_{p}^{*}(g, w, t)$ will be defined in Section 6.

We set

$$
\begin{aligned}
& T^{\langle j\rangle}:=\prod_{i=1}^{j} T_{i}^{1 / 4}, \quad \mathbb{R}_{(j)}:=\left\{x_{j} \in \mathbb{R}\right\}, \\
& \mathbb{R}_{\leq j}^{j}:=\left\{\left(x_{1}, \cdots, x_{j}\right) \in \mathbb{R}^{j}\right\}, \quad \mathbb{R}_{j \leq}^{s-j+1}:=\left\{\left(x_{j}, \cdots, x_{s}\right) \in \mathbb{R}^{s-j+1}\right\}, \\
& \mathbb{R}_{j}^{s-1}:=\left\{\left(x_{1}, \cdots, x_{j-1}, x_{j+1}, \cdots, x_{s}\right) \in \mathbb{R}^{s-1}\right\}, \\
& W:=\prod_{i=1}^{s} w_{i}, W_{j}:=\prod_{i=1, i \neq j}^{s} w_{i}, \quad j=1,2, \cdots, s .
\end{aligned}
$$

We need the infinite-finite inequality.

Theorem 3.7 (Infinite-finite inequality). Let $0<p \leq \infty, L>0$, and let

$$
\begin{aligned}
P(X) \in \mathcal{P}_{n, \cdots, n}\left(\mathbb{R}^{s}\right)\left(=: \mathcal{P}_{n ; s}\left(\mathbb{R}^{s}\right)\right) \text {. Then } \\
\\
\quad\|W(X) P(X)\|_{L^{p}\left(\mathbb{R}^{s}\right)} \leq C\|W(X) P(X)\|_{L^{p}\left(\left|x_{i}\right| \leq a_{n}^{(i)}\left(1-L \delta_{n}^{(i)}\right), i=1,2, \cdots, s\right)} .
\end{aligned}
$$

If $r>1$, then there exists $\varepsilon>0$ such that

$$
\|W(X) P(X)\|_{L^{p}\left(\mathbb{R}_{, r}^{s}\right)} \leq C \exp \left(-n^{\varepsilon}\right)\|W(X) P(X)\|_{L^{p}\left(\mathbb{R}^{s}\right)},
$$

where $\mathbb{R}_{i, r}^{s}:=\left\{x_{i} ;\left|x_{i}\right| \geq a_{r n}^{(i)}\right\} \times \mathbb{R}_{i}^{s-1}$.

To prove Theorem 3.7 we use the following proposition with $s=1$.

Proposition 3.8 ([2], Theorem 1.9). Let $0<p \leq \infty, L>0$, and let $P(x) \in \mathcal{P}_{n}(\mathbb{R})$. Then

$$
\|w(x) P(x)\|_{L^{p}(\mathbb{R})} \leq C\|w(x) P(x)\|_{L^{p}\left(|x| \leq a_{n}\left(1-L \delta_{n}\right)\right)} .
$$

If $r>1$, then there exists $\varepsilon>0$ such that

$$
\|w(x) P(x)\|_{L^{p}\left(a_{r n} \leq x \mid\right)} \leq C \exp \left(-n^{\varepsilon}\right)\|w(x) P(x)\|_{L^{p}\left(|x| \leq a_{n}\right)} .
$$

Proof of Theorem 3.7. For the proof of (3.12) we use (3.14). We put $A$ for the left side of the above equation. Let $0<p<\infty$. By repeatedly applying Proposition 
3.8 (3.14), we have

$$
\begin{aligned}
A^{p}= & \int_{-\infty}^{\infty} \cdots \int_{-\infty}^{\infty}\left|w_{2}\left(x_{2}\right) \cdots w_{s}\left(x_{s}\right)\right|^{p} \times\left\{\int_{-\infty}^{\infty}\left|w_{1}\left(x_{1}\right) P\left(x_{1}, \cdots, x_{s}\right)\right|^{p} \mathrm{~d} x_{1}\right\} \mathrm{d} x_{2} \cdots \mathrm{d} x_{s} \\
\leq & C_{1} \int_{-\infty}^{\infty} \cdots \int_{-\infty}^{\infty}\left|w_{2}\left(x_{2}\right) \cdots w_{s}\left(x_{s}\right)\right|^{p} \int_{-a_{n}^{(1)}\left(1-L \delta_{n}^{(1)}\right)}^{a_{1}^{(1)}\left(1-L \delta_{n}^{(1)}\right)}\left|w_{1}\left(x_{1}\right) P\left(x_{1}, \cdots, x_{s}\right)\right|^{p} \mathrm{~d} x_{1} \cdots \mathrm{d} x_{s} \\
= & C_{1} \int_{-a_{n}^{(1)}\left(1-L \delta_{n}^{(1)}\right)}^{a_{n}^{(1)}\left(1-L \delta_{n}^{(1)}\right)} w_{1}^{p}\left(x_{1}\right) \int_{-\infty}^{\infty} \cdots \int_{-\infty}^{\infty}\left|w_{2}\left(x_{2}\right) \cdots w_{s}\left(x_{s}\right) P\left(x_{1}, \cdots, x_{s}\right)\right|^{p} \mathrm{~d} x_{2} \cdots \mathrm{d} x_{s} \mathrm{~d} x_{1} \\
\leq & C_{2} \int_{-a_{n}^{(1)}\left(1-L \delta_{n}^{(1)}\right)}^{a_{n}^{(1)}\left(1-L \delta_{n}^{(1)}\right)} \int_{-a_{n}^{(2)}\left(1-L \delta_{n}^{(2)}\right)}^{a_{n}^{(2)}\left(1-L \delta_{1}^{(2)}\right)} w_{1}^{p}\left(x_{1}\right) w_{2}^{p}\left(x_{2}\right) \\
& \times \int_{-\infty}^{\infty} \cdots \int_{-\infty}^{\infty}\left|w_{3}\left(x_{2}\right) \cdots w_{s}\left(x_{s}\right) P\left(x_{1}, \cdots, x_{s}\right)\right|^{p} \mathrm{~d} x_{3} \cdots \mathrm{d} x_{d} \mathrm{~d} x_{1} \mathrm{~d} x_{2} \\
\leq & \cdots \\
\leq & C_{s} \int_{-a_{n}^{(1)}\left(1-L \delta_{n}^{(1)}\right)}^{a_{n}^{(1)}\left(1-L \delta_{n}^{(1)}\right)} \cdots \int_{-a_{n}^{(s)}\left(1-L \delta_{n}^{(s)}\right)}^{a_{a}^{(s)}\left(1-L \delta_{n}^{(s)}\right)}\left|w_{1}\left(x_{1}\right) \cdots w_{s}\left(x_{s}\right) P\left(x_{1}, \cdots, x_{s}\right)\right|^{p} \mathrm{~d} x_{1} \cdots \mathrm{d} x_{s} \\
= & C_{s}\|W(X) P(X)\|_{L^{p}\left(\left|x_{i}\right| \leq a_{n}^{(i)}\left(1-L \delta_{n}^{(i)}\right), i=1,2, \cdots, s\right)}^{p}
\end{aligned}
$$

Next, we show the case of $p=\infty$.

$$
\begin{aligned}
A= & \sup _{x_{s} \in \mathbb{R}} \cdots \sup _{x_{2} \in \mathbb{R}}\left|w_{2}\left(x_{2}\right) \cdots w_{s}\left(x_{s}\right)\right| \sup _{x_{1} \in \mathbb{R}}\left|w_{1}\left(x_{1}\right) P\left(x_{1}, \cdots, x_{s}\right)\right| \\
\leq & C_{1} \sup _{x_{s} \in \mathbb{R}} \cdots \sup _{x_{2} \in \mathbb{R}}\left|w_{2}\left(x_{2}\right) \cdots w_{s}\left(x_{s}\right)\right| \sup _{\left|x_{1}\right| \leq a_{n}^{(1)}\left(1-L \delta_{n}^{(1)}\right)}\left|w_{1}\left(x_{1}\right) P\left(x_{1}, \cdots, x_{s}\right)\right| \\
= & C_{1} \sup _{x_{s} \in \mathbb{R}} \cdots \sup _{x_{3} \in \mathbb{R}}\left|w_{3}\left(x_{3}\right) \cdots w_{s}\left(x_{s}\right)\right| \sup _{\left|x_{1}\right| \leq a_{n}^{(1)}\left(1-L \delta_{n}^{(1)}\right)} \sup _{x_{2} \in \mathbb{R}}\left|w_{1}\left(x_{1}\right) w_{2}\left(x_{2}\right) P\left(x_{1}, \cdots, x_{s}\right)\right| \\
\leq & C_{1} C_{2} \sup _{x_{s} \in \mathbb{R}} \cdots \sup \mid x_{3} \in \mathbb{R} \\
& \times \sup _{\left|x_{1}\right| \leq a_{n}^{(1)}\left(1-L \delta_{n}^{(1)}\right)\left|x_{2}\right| \leq a_{n}^{(2)}\left(1-L \delta_{n}^{(2)}\right)}\left|w_{1}\left(x_{1}\right) \cdots w_{s}\left(x_{s}\right)\right| \\
\leq & \cdots \quad w_{2}\left(x_{2}\right) P\left(x_{1}, \cdots, x_{s}\right) \mid \\
\leq & C_{1} C_{2} \cdots C_{s} \sup _{\left|x_{1}\right| \leq a_{n}^{(1)}\left(1-L \delta_{n}^{(1)}\right)\left|x_{2}\right| \leq a_{n}^{(2)}\left(1-L \delta_{n}^{(2)}\right)} \quad \sup _{\left|x_{2}\right| \leq a_{n}^{(s)}\left(1-L \delta_{n}^{(s)}\right)} \mid w_{1}\left(x_{1}\right) w_{2}\left(x_{2}\right) \\
& \times \cdots \times w_{s}\left(x_{s}\right) P\left(x_{1}, \cdots, x_{s}\right) \mid \\
& C \quad \sup _{\left|x_{i}\right| \leq a_{n}^{(i)}\left(1-L \delta_{n}^{(i)}\right), i=1, \cdots, s}\left|w_{1}\left(x_{1}\right) w_{2}\left(x_{2}\right) \cdots w_{s}\left(x_{s}\right) P\left(x_{1}, \cdots, x_{s}\right)\right| .
\end{aligned}
$$

Similarly, using Proposition 3.8 (3.15), we easily have (3.13). \# Lemma 3.9. Let $1 \leq p \leq \infty$.

(1) We assume that $w_{i} \in \mathcal{F}\left(C^{2}+\right), i=1,2, \cdots, s$ satisfies (3.6). Then there exists a constant $C>0$ such that when $W h \in L_{p}\left(\mathbb{R}^{s}\right)$, 


$$
\left\|\frac{W v_{n}^{[j]}(h)}{T^{\langle j\rangle}}\right\|_{L^{p}\left(\mathbb{R}^{s}\right)} \leq C\|W h\|_{L^{p}\left(\mathbb{R}^{s}\right)}, j=1,2, \cdots, s,
$$

and

$$
\left\|W v_{n}^{[s]}(f)\right\|_{L^{p}\left(\mathbb{R}^{s}\right)} \leq C \prod_{i=1}^{s} T_{i}^{1 / 4}\left(a_{n}^{(i)}\right)\|W f\|_{L^{p}\left(\mathbb{R}^{s}\right)} .
$$

(2) We assume that $w_{i} \in \mathcal{F}_{\lambda}\left(C^{3}+\right)(0<\lambda<3 / 2), i=1,2, \cdots, s$. Let $T^{\langle s\rangle} W h \in L^{p}\left(\mathbb{R}^{s}\right)$, then

$$
\left\|W v_{n}^{[j]}(h)\right\|_{L^{p}\left(\mathbb{R}^{s}\right)} \leq C\left\|T^{\langle j\rangle} W h\right\|_{L^{p}\left(\mathbb{R}^{s}\right)}, j=1,2, \cdots, s .
$$

Proof. (1) From Theorem 3.4 (1), for $j=1$

$$
\left\|\frac{W v_{n}^{[1]}(h)}{T^{(1\rangle}}\right\|_{L^{p}\left(\mathbb{R}^{s}\right)}=\left\|\frac{W v_{n, 1}\left(h_{\hat{X}_{1}}\right)}{T_{1}^{1 / 4}}\right\|_{L^{p}\left(\mathbb{R}_{\leq 1}^{1}\right)}\left\|_{L^{p}\left(\mathbb{R}_{2 \varsigma}^{s-1}\right)} \leq C\right\| W h \|_{L^{p}\left(\mathbb{R}^{s}\right)} .
$$

Inductively,

$$
\begin{aligned}
& \left\|\frac{W v_{n}^{[j]}(h)}{T^{\langle j\rangle}}\right\|_{L^{p}\left(\mathbb{R}^{s}\right)}=\left\|\frac{W v_{n}^{[j-1]}\left(v_{n, j}(h)\right)}{T^{\langle j\rangle}}\right\|_{L^{p}\left(\mathbb{R}_{s j-1}^{j j-1}\right)} \|_{L^{p}\left(\mathbb{R}_{j \leq}^{s j j+1}\right)} \\
& \leq C\left\|\frac{W v_{n, j}\left(h_{\hat{X}_{j}}\right)}{T_{j}^{1 / 4}}\right\|_{L^{p}\left(\mathbb{R}^{s}\right)} \leq C\|W h\|_{L^{p}\left(\mathbb{R}^{s}\right)} .
\end{aligned}
$$

For the second formula, using Theorem 3.7 and the above inequality, we have

$$
\begin{aligned}
& \left\|W v_{n}^{[j]}(h)\right\|_{L^{p}\left(\mathbb{R}^{s}\right)} \\
& \leq C \prod_{i=1}^{j} T_{i}^{1 / 4}\left(a_{n}^{(i)}\right)\left\|\left(\prod_{k=j+1}^{s} w_{k}\right)\right\|\left(\frac{\left(\prod_{i=1}^{j} w_{i}\right) v_{n}^{[j]}(h)}{T^{\langle j\rangle}}\left\|_{L^{p}\left(\left|x_{i}\right| \leq a_{2 n}^{(i)}\right), 1 \leq i \leq j}\right\|_{L^{p}\left(\mathbb{R}_{j+1 \leq 1}^{s-j}\right)}\right. \\
& \leq C \prod_{i=1}^{j} T_{i}^{1 / 4}\left(a_{n}^{(i)}\right)\|W h\|_{L^{p}\left(\mathbb{R}^{s}\right)}, \\
& j=1,2, \cdots, s .
\end{aligned}
$$

Similarly we have the following:

(2) From Theorem 3.4 (2) for $j=1$,

$$
\begin{aligned}
& \left\|W v_{n}^{[1]}(h)\right\|_{L^{p}\left(\mathbb{R}^{s}\right)}=\left\|W v_{n, 1}(h)\right\|_{L^{p}\left(\mathbb{R}^{s}\right)} \leq C\left\|W T_{1}^{1 / 4} h\right\|_{L^{p}\left(\mathbb{R}^{s}\right)}, \\
& j=1,2, \cdots, s .
\end{aligned}
$$

Inductively, 


$$
\begin{aligned}
\left\|W v_{n}^{[j]}(h)\right\|_{L^{p}\left(\mathbb{R}^{s}\right)} & =\left\|W v_{n}^{[j-1]}\left(v_{n, j}(h)\right)\right\|_{L^{p}\left(\mathbb{R}^{s}\right)} \leq C\left\|W T^{\langle j-1\rangle} v_{n, j}(h)\right\|_{L^{p}\left(\mathbb{R}^{s}\right)} \\
& \leq C\left\|W T^{\langle j\rangle} h\right\|_{L^{p}\left(\mathbb{R}^{s}\right)} .
\end{aligned}
$$

Proof of (3.7) in Theorem 3.3. By Proposition 3.5 (2) and Proposition 3.6, we get

$$
\left\|w_{1}(x)\left(f_{\hat{X}_{1}}-v_{n, 1}\left(f_{\hat{X}_{1}}\right)\right)\right\|_{L^{p}(\mathbb{R})} \leq C E_{n, p}\left(f_{\hat{X}_{1}} ; T_{1}^{1 / 4} w_{1}\right) \leq C_{1} \bar{\omega}_{p, 1}\left(f_{\hat{X}_{1}}, T_{1}^{1 / 4} w_{1} ; c_{1} \frac{a_{n}^{(1)}}{n}\right),
$$

where the constant $C_{1}$ and $c_{1}$ is independent of $\hat{X}_{1}$. Similarly, for $j=1,2, \cdots, s$,

$$
\left\|w_{j}(x)\left(f_{\hat{X}_{j}}-v_{n, j}\left(f_{\hat{X}_{j}}\right)\right)\right\|_{L^{p}(\mathbb{R})} \leq C_{j} \bar{\omega}_{p, j}\left(f_{\hat{X}_{j}}, T_{j}^{1 / 4} w_{j} ; c_{j} \frac{a_{n}^{(j)}}{n}\right) .
$$

Using $\quad f-v_{n}^{[s]}=\left(f-v_{n}^{[1]}(f)\right)+\left(v_{n}^{[1]}(f)-v_{n}^{[2]}(f)\right)+\cdots+\left(v_{n}^{[s-1]}(f)-v_{n}^{[s]}(f)\right)$, we get from Lemma 3.9 (2) and (3.2),

$$
\begin{aligned}
& \left\|W\left(f-v_{n}^{[s]}(f)\right)\right\|_{L^{p}\left(\mathbb{R}^{s}\right)} \\
& \leq\left\|W\left(f-v_{n}^{[1]}(f)\right)\right\|_{L^{p}\left(\mathbb{R}^{s}\right)}+\sum_{j=2}^{s}\left\|W\left(v_{n}^{[j-1]}(f)-v_{n}^{[j]}(f)\right)\right\|_{L^{p}\left(\mathbb{R}^{s}\right)} \\
& \leq C\left[\left\|W\left(f-v_{n, 1}(f)\right)\right\|_{L^{p}\left(\mathbb{R}^{s}\right)}+\sum_{j=2}^{s}\left\|W v_{n}^{[j-1]}\left(f-v_{n, j}(f)\right)\right\|_{\left.L^{p}\left(\mathbb{R}^{s}\right)\right]}\right] \\
& \leq C\left[\left\|W\left(f-v_{n, 1}(f)\right)\right\|_{L^{p}\left(\mathbb{R}^{s}\right)}+\sum_{j=2}^{s}\left\|W\left(\prod_{k=1}^{j-1} T_{k}^{1 / 4}\right)\left(f-v_{n, j}(f)\right)\right\|_{\left.L^{p}\left(\mathbb{R}^{s}\right)\right]}\right] \\
& \quad C_{1}\left\|W_{1} \bar{\omega}_{p, 1}\left(f_{\hat{X}_{1}}, T_{1}^{1 / 4} w_{1} ; c_{1} \frac{a_{n}^{(1)}}{n}\right)\right\|\left\|_{L^{p}\left(\mathbb{R}_{1}^{s-1}\right)} \sum_{j=2}^{s} C_{j}\right\| W_{j}\left(\prod_{k=1}^{j-1} T_{k}^{1 / 4}\right) \bar{\omega}_{p, j}\left(f_{\hat{X}_{j}}, T_{j}^{1 / 4} w_{j} ; c_{j} \frac{a_{n}^{(j)}}{n}\right) \|_{L^{p}\left(\mathbb{R}_{j}^{s-1}\right)}
\end{aligned}
$$

by (3.16)

$$
\leq \sum_{j=1}^{s} C_{j}\left\|W_{j}\left(\prod_{k=1}^{j-1} T_{k}^{1 / 4}\right) \bar{\omega}_{p, j}\left(f_{\hat{X}_{j}}, T_{j}^{1 / 4} w_{j} ; c_{j} \frac{a_{n}^{(j)}}{n}\right)\right\|_{L^{p}\left(\mathbb{R}_{j}^{s-1}\right)},
$$

where $\prod_{k=1}^{j-1} T_{k}^{1 / 4}=1$ for $j=1$. Hence we obtain (3.7).

Proof of (3.10) in Theorem 3.3. By Proposition 3.5 (1) and Proposition 3.6, we get

$$
\left\|\frac{w_{1}(x)\left(f_{\hat{X}_{1}}-v_{n, 1}\left(f_{\hat{X}_{1}}\right)\right)}{T_{1}^{1 / 4}(x)}\right\|_{L^{p}(\mathbb{R})} \leq C E_{n, p}\left(f_{\hat{X}_{1}} ; w_{1}\right) \leq C_{1} \bar{\omega}_{p, 1}\left(f_{\hat{X}_{1}}, w_{1} ; c_{1} \frac{a_{n}^{(1)}}{n}\right),
$$

where the constant $C_{1}$ and $c_{1}$ is independent of $\hat{X}_{1}$. Similarly, for $j=1,2, \cdots, s$, 


$$
\left\|\frac{w_{j}(x)\left(f_{\hat{X}_{j}}-v_{n, j}\left(f_{\hat{X}_{j}}\right)\right)}{T_{j}^{1 / 4}(x)}\right\|_{L^{p}(\mathbb{R})} \leq C_{j} \bar{\omega}_{p, j}\left(f_{\hat{X}_{j}}, w_{j} ; c_{j} \frac{a_{n}^{(j)}}{n}\right) .
$$

Using $f-v_{n}^{[s]}=\left(f-v_{n}^{[1]}(f)\right)+\left(v_{n}^{[1]}(f)-v_{n}^{[2]}(f)\right)+\cdots+\left(v_{n}^{[s-1]}(f)-v_{n}^{[s]}(f)\right)$, we get from Lemma 3.9 (1) and (3.2),

$$
\begin{aligned}
& \left\|\frac{W\left(f-v_{n}^{[s]}(f)\right)}{T^{\langle s\rangle}}\right\|_{L^{p}\left(\mathbb{R}^{s}\right)} \\
& \leq\left\|\frac{W\left(f-v_{n}^{[1]}(f)\right)}{T^{\langle 1\rangle}}\right\|_{L^{p}\left(\mathbb{R}^{s}\right)}+\sum_{j=2}^{s}\left\|\frac{W\left(v_{n}^{[j-1]}(f)-v_{n}^{[j]}(f)\right)}{T^{\langle j\rangle}}\right\|_{L^{p}\left(\mathbb{R}^{s}\right)} \\
& \leq C\left[\left\|\frac{W\left(f-v_{n, 1}(f)\right)}{T_{1}^{1 / 4}}\right\|_{L^{p}\left(\mathbb{R}^{s}\right)}+\sum_{j=2}^{s}\left\|\frac{W v_{n}^{[j-1]}\left(\left\{f-v_{n, j}(f)\right\}\right) / T_{j}^{1 / 4}}{T^{\langle j-1\rangle}}\right\|_{L^{p}\left(\mathbb{R}^{s}\right)}\right] \\
& \leq C\left[\left\|\frac{W\left(f-v_{n, 1}(f)\right)}{T_{1}^{1 / 4}}\right\|_{L^{p}\left(\mathbb{R}^{s}\right)}+\sum_{j=2}^{s}\left\|\frac{W\left(f-v_{n, j}(f)\right)}{T_{j}^{1 / 4}}\right\|_{L^{p}\left(\mathbb{R}^{s}\right)}\right] \\
& \leq C_{1}\left\|\left(\prod_{2 \leq i \leq s} w_{i}\right) \bar{\omega}_{p, 1}\left(f_{\hat{X}_{1}}, w_{1} ; c_{1} \frac{a_{n}^{(1)}}{n}\right)\right\|_{L^{p}\left(\mathbb{R}_{1}^{s-1}\right)} \\
& +\sum_{j=2}^{s} C_{j}\left\|\left(\prod_{1 \leq i \leq s, i \neq j} w_{i}\right) \bar{\omega}_{p, j}\left(f_{\hat{X}_{j}}, w_{j} ; c_{j} \frac{a_{n}^{(j)}}{n}\right)\right\|_{L^{p}\left(\mathbb{R}_{j}^{s-1}\right)}
\end{aligned}
$$

by (3.17)

$$
\leq \sum_{j=1}^{s} C_{j}\left\|\left(\prod_{1 \leq i \leq s, i \neq j} w_{i}\right) \bar{\omega}_{p, j}\left(f_{\hat{X}_{j}}, w_{j} ; c_{j} \frac{a_{n}^{(j)}}{n}\right)\right\|_{L^{p}\left(\mathbb{R}_{j}^{s-1}\right)} .
$$

Hence we obtain (3.10).

To prove (3.9) and (3.11) we need a lemma:

Lemma 3.10. Let $0<h \leq 1, \sigma(t) \geq 1$ and $|x| \leq \sigma(2 t)$. We have

$$
\left\|(w f)\left(x \pm h \Phi_{t}(x)\right)\right\|_{L^{p}(\mathbb{R})} \sim\|(w f)(x)\|_{L^{p}(\mathbb{R})} .
$$

Proof. We may show

$$
\left\|(w f)\left(x \pm h \sqrt{1-\frac{|x|}{\sigma(t)}}\right)\right\|_{L^{p}(\mathbb{R})} \sim\|(w f)(x)\|_{L^{p}(\mathbb{R})} .
$$

Let $x>0$. If we put

$$
x \pm h \sqrt{1-\frac{x}{\sigma(t)}}=: y, \frac{a_{2 u}}{2 u}=t, \frac{a_{v}}{v}=2 t,
$$


we will see

$$
\frac{1}{2} \leq \frac{\mathrm{d} y}{\mathrm{~d} x} \leq \frac{3}{2}
$$

Then we conclude (3.19). Now, from (3.20)

$$
\frac{\mathrm{d} y}{\mathrm{~d} x}=1 \mp \frac{h}{2 \sqrt{\sigma(t)} \sqrt{\sigma(t)-x}} .
$$

Since $a_{2 u} / u=2 t$, we see

$$
\frac{a_{v}}{v}=\frac{a_{2 u}}{u}>\frac{a_{u}}{u}
$$

that is, we have

$$
\sigma(2 t)=a_{v}<a_{u}<\sigma(t)=a_{2 u} \text {. }
$$

Then, using ([2], Lemmas 3.6, 3.7), we see

$$
\begin{aligned}
\frac{h}{2 \sqrt{\sigma(t)} \sqrt{\sigma(t)-x}} & \leq \frac{t}{2 \sqrt{\sigma(t)} \sqrt{\sigma(t)-\sigma(2 t)}} \leq \frac{\sqrt{a_{2 u}}}{4 u} \frac{1}{\sqrt{a_{2 u}-a_{u}}} \\
& \leq \frac{\sqrt{a_{2 u}}}{4 u} \sqrt{C_{1} \frac{T\left(a_{2 u}\right)}{a_{2 u}}} \leq \frac{1}{4 u} \sqrt{C_{1} C_{2}} u^{1-\delta}=\frac{\sqrt{C_{1} C_{2}}}{4} \frac{1}{u^{\delta}} \leq \frac{1}{2}
\end{aligned}
$$

for some $0<\delta \leq 1$ and $u$ large enough. We have (3.21). So we conclude (3.19).

Proof of (3.9). We will estimate

$$
\left\|W_{j} T^{\langle j-1\rangle}\left\{\frac{1}{t} \int_{0}^{t}\left\|T_{j}^{1 / 4}(x) w_{j}(x)\left(\Delta_{h \Phi_{t, j}(x)} f_{\hat{X}_{j}}(x)\right)\right\|_{L^{p}\left(|x| \leq \sigma_{j}(2 t)\right)}^{p} \mathrm{~d} h\right\}^{1 / p}\right\|_{L^{p}\left(\mathbb{R}_{j}^{s-1}\right)} .
$$

To do so we may estimate

$$
I_{1}:=\left\{\frac{1}{t} \int_{0}^{t}\left\|T_{j}^{1 / 4}(x) w_{j}(x)\left(\Delta_{h \Phi_{t, j}(x)} f_{\hat{X}_{j}}(x)\right)\right\|_{L^{p}\left(|x| \leq \sigma_{j}(2 t)\right)}^{p} \mathrm{~d} h\right\}^{1 / p} .
$$

For $\varepsilon>0$ we take $K>0$ large enough, and then by $f \in L_{T^{\langle s\rangle_{w}}}^{p^{*}}(\mathbb{R})$ we can select a continuous function $f_{K}$ such that

$$
\begin{aligned}
I_{1} \leq & \left\{\frac{1}{t} \int_{0}^{t}\left\|T_{j}^{1 / 4}(x) w_{j}(x)\left(\Delta_{h \Phi_{t, j}(x)}\left(f-f_{K}\right)_{\hat{X}_{j}}(x)\right)\right\|_{L^{p}\left(|x| \leq \sigma_{j}(2 t)\right)}^{p} \mathrm{~d} h\right\}^{1 / p} \\
& +\left\{\frac{1}{t} \int_{0}^{t}\left\|T_{j}^{1 / 4}(x) w_{j}(x)\left(\Delta_{h \Phi_{t, j}(x)}\left(f_{K}\right)_{\hat{X}_{j}}(x)\right)\right\|_{L^{p}\left(|x| \leq \sigma_{j}(2 t)\right)}^{p} \mathrm{~d} h\right\}^{1 / p} \\
= & A+B .
\end{aligned}
$$

We note $\quad w_{j}(x) \sim w\left(x+(h / 2) \Phi_{t, j}(x)\right) \sim w\left(x-(h / 2) \Phi_{t, j}(x)\right) \quad$ (see [7], Lemma 7). If $\sigma_{j}(2 t) \leq K$ from our assumption and Lemma 3.10 we have 


$$
\begin{aligned}
A & \leq C\left\{\frac{1}{t} \int_{0}^{t}\left\|T_{j}^{1 / 4}(x) w_{j}(x)\left(f-f_{K}\right)_{\hat{X}_{j}}(x)\right\|_{L^{p}\left(|x| \leq \sigma_{j}(2 t)\right)}^{p} \mathrm{~d} h\right\}^{1 / p} \\
& \leq C \varepsilon\left\{\frac{1}{t} \int_{0}^{t} \mathrm{~d} h\right\}^{1 / p} \leq C \varepsilon .
\end{aligned}
$$

If $\sigma_{j}(2 t)>K$, then by Lemma 3.10 we see

$$
\begin{aligned}
A \leq & C\left\{\frac{1}{t} \int_{0}^{t}\left\|T_{j}^{1 / 4}(x) w_{j}(x)\left(f-f_{K}\right)_{\hat{X}_{j}}(x)\right\|_{L^{p}(|x| \leq K)}^{p} \mathrm{~d} h\right\}^{1 / p} \\
& +\left\{\frac{1}{t} \int_{0}^{t}\left\|T_{j}^{1 / 4}(x) w_{j}(x) f_{\hat{X}_{j}}(x)\right\|_{L^{p}\left(K<|x| \leq \sigma_{j}(2 t)\right)}^{p} \mathrm{~d} h\right\}^{1 / p} \\
\leq & C \varepsilon+C_{1} \varepsilon \leq C_{2} \varepsilon .
\end{aligned}
$$

When we take $t>0$ small enough, we see

$$
\begin{aligned}
B & =\left\{\frac{1}{t} \int_{0}^{t}\left\|T_{j}^{1 / 4}(x) w_{j}(x)\left(\Delta_{h \Phi_{t, j}(x)}\left(f_{K}\right)_{\hat{X}_{j}}(x)\right)\right\|_{L^{p}(|x| \leq K)}^{p} \mathrm{~d} h\right\}^{1 / p} \\
& \leq\left\{\frac{1}{t} \int_{0}^{t} \varepsilon \mathrm{d} h\right\}^{1 / p} \leq C \varepsilon,
\end{aligned}
$$

because of the continuity of $f_{K}$. Therefore we have $I_{1}<\varepsilon$. Consequently, we have

$$
\begin{aligned}
& \left\|W_{j} T^{\langle j-1\rangle}\left\{\frac{1}{t} \int_{0}^{t}\left\|T_{j}^{1 / 4}(x) w_{j}(x)\left(\Delta_{h \Phi_{t, j}(x)} f_{\hat{X}_{j}}(x)\right)\right\|_{L^{p}\left(|x| \leq \sigma_{j}(2 t)\right)}^{p} \mathrm{~d} h\right\}^{1 / p}\right\|_{L^{p}\left(\mathbb{R}_{j}^{s-1}\right)} \\
& \leq C \varepsilon\left\|W_{j} T^{\langle j-1\rangle}\right\|_{L^{p}\left(\mathbb{R}_{j}^{s-1}\right)} \leq C \varepsilon .
\end{aligned}
$$

Finally, we see

$$
\begin{aligned}
& \left\|W_{j} T^{\langle j-1\rangle} \inf _{c_{j}(\text { constant })}\right\|\left(f_{\hat{X}_{j}}(x)-c_{j}\right) T_{j}^{1 / 4}(x) w_{j}(x)\left\|_{\left.L^{p}\left(\mathbb{R}\left[-\sigma_{j}(4 t), \sigma_{j}(4 t)\right]\right]\right)}\right\|_{L^{p}\left(\mathbb{R}_{j}^{s-1}\right)} \\
& \leq C\left\|W_{j} T^{\langle j-1\rangle}\right\| f_{\hat{X}_{j}}(x) T_{j}^{1 / 4}(x) w_{j}(x)\left\|_{\left.L^{p}\left(\mathbb{R}\left[-\sigma_{j}(4 t), \sigma_{j}(4 t)\right]\right]\right)}\right\|_{L^{p}\left(\mathbb{R}_{j}^{s-1}\right)} \\
& \leq C w_{j}^{1 / 4}\left(\sigma_{j}(4 t)\right)\|W f\|_{L^{p}\left(\mathbb{R}_{j}^{s}\right)^{s}} .
\end{aligned}
$$

Here, if we set $4 t=a_{u} / u$, then we see

$$
w_{j}^{1 / 4}\left(\sigma_{j}(4 t)\right)=\exp \left(-\frac{1}{4} Q_{j}\left(a_{u}\right)\right) \sim \exp \left(-\frac{u}{2 \sqrt{T\left(a_{u}\right)}}\right) \leq \mathrm{e}^{-u^{\delta}}
$$

for some $0<\delta<1$, that is,

$$
w_{j}^{1 / 4}\left(\sigma_{j}(4 t)\right) \leq C \mathrm{e}^{-u^{\delta}} \leq C \frac{a_{u}}{4 u}=C t .
$$

Therefore 


$$
\begin{aligned}
& \left\|W_{j} T^{\langle j-1\rangle} \inf _{c_{j} \text { (constant) }}\right\|\left(f_{\hat{X}_{j}}(x)-c_{j}\right) T_{j}^{1 / 4}(x) w_{j}(x)\left\|_{L^{p}\left(\mathbb{R} \backslash\left[-\sigma_{j}(4 t), \sigma_{j}(4 t)\right]\right)}\right\|_{L^{p}\left(\mathbb{R}_{j}^{s-1}\right)} \\
& \leq C t .
\end{aligned}
$$

For given $\varepsilon>0$ if we take $K>0$ large enough and then $t>0$ small enough, then by (3.22) and (3.23) we have

$$
\left\|W_{j} T^{\langle j-1\rangle} \bar{\omega}_{p, j}\left(f_{\hat{X}_{j}}, T_{j}^{1 / 4} w_{j}, t\right)\right\|_{L^{p}\left(\mathbb{R}_{j}^{s-1}\right)}<\varepsilon .
$$

Consequently, we have (3.9).

Proof of (3.11). If in the proof of (3.9) we set as $T=1$ (constant), then we obtain (3.11). \#

Corollary 3.11. We suppose that $w_{j}=\exp \left(-Q_{j}\right) \in \mathcal{F}\left(C^{2}+\right), j=1,2, \cdots, s$ are the Freud-type weights. Let $1 \leq p \leq \infty$. Then we have

$$
\begin{aligned}
& \left\|W\left(f-v_{n}^{[s]}(f)\right)\right\|_{L^{p}\left(\mathbb{R}^{s}\right)} \\
& \leq \sum_{j=1}^{s} C_{j}\left\|\left(\prod_{1 \leq i \leq s, i \neq j} w_{i}\right) \bar{\omega}_{p, j}\left(f_{\hat{X}_{j}}, w_{j} ; c_{j} \frac{a_{n}^{(j)}}{n}\right)\right\|_{L^{p}\left(\mathbb{R}_{j}^{s-1}\right)} .
\end{aligned}
$$

Especially $f \in L_{w}^{p^{*}}\left(\mathbb{R}^{s}\right)$, then we have

$$
\sum_{j=1}^{s} C_{j}\left\|\left(\prod_{1 \leq i \leq s, i \neq j} w_{i}\right) \bar{\omega}_{p, j}\left(f_{\hat{X}_{j}}, w_{j} ; C_{j} \frac{a_{n}^{(j)}}{n}\right)\right\|_{L^{p}\left(\mathbb{R}_{j}^{s-1}\right)} \rightarrow 0 \text { as } n \rightarrow \infty .
$$

Corollary 3.12. We suppose $w_{j}=\exp \left(-Q_{j}\right) \in \mathcal{F}_{\lambda}\left(C^{3}+\right)(0<\lambda<3 / 2), j=1,2, \cdots, s$, and let (3.6) hold. Let $1 \leq p \leq \infty$. If $\left.T^{\langle s\rangle} W f \in C_{0}\left(\mathbb{R}^{s}\right) \cap L^{p}\left(\mathbb{R}^{s}\right)\right)$, then we have

$$
\left\|W\left(f-v_{n}^{[s]}(f)\right)\right\|_{L^{p}\left(\mathbb{R}^{s}\right)} \rightarrow 0 \text { as } n \rightarrow \infty .
$$

Moreover, we suppose $w_{j}=\exp \left(-Q_{j}\right) \in \mathcal{F}\left(C^{2}+\right), j=1,2, \cdots, s$, and let (3.6) hold. If $W f \in C_{0}\left(\mathbb{R}^{s}\right) \cap L^{p}\left(\mathbb{R}^{s}\right)$, then we have

$$
\left\|\frac{W\left(f-v_{n}^{[s]}(f)\right)}{T^{\langle s\rangle}}\right\|_{\left.L^{p} \mathbb{R}^{s}\right)} \rightarrow 0 \text { as } n \rightarrow \infty \text {. }
$$

\section{A Property of Higher Order Derivatives}

In this section we show an important theorem which is useful in approximation theory. We use the following notations for $w_{i}\left(x_{i}\right)=\exp \left(-Q_{i}\left(x_{i}\right)\right) \in \mathcal{F}\left(C^{2}+\right), i=1,2, \cdots, s$. Let $r$ be a positive integer, and $\left|x_{i}\right| \geq \gamma>0$.

$$
W_{0}:=\prod_{i=1}^{s} w_{i, 0} ; Q_{i}^{\prime}\left(x_{i}\right)^{r} w_{i}\left(x_{i}\right) \sim w_{i, 0}\left(x_{i}\right)=\exp \left(-Q_{i, 0}\left(x_{i}\right)\right) \in \mathcal{F}\left(C^{2}+\right),
$$




$$
\begin{aligned}
& W_{v}:=\prod_{i=1}^{s} w_{i, v} ; Q_{i}^{\prime}\left(x_{i}\right)^{r-v} w_{i}\left(x_{i}\right) \sim w_{i, v}\left(x_{i}\right)=\exp \left(-Q_{i, v}\left(x_{i}\right)\right) \in \mathcal{F}\left(C^{2}+\right), \\
& v=1,2, \cdots, r .
\end{aligned}
$$

Then we see

$$
Q_{i}^{\prime}\left(x_{i}\right)^{r-v+1} w_{i}\left(x_{i}\right) \sim Q_{i, v}^{\prime}\left(x_{i}\right) w_{i, v}\left(x_{i}\right) .
$$

Especially, if $v=r$, then

$$
w_{i, r}\left(x_{i}\right)=w_{i}\left(x_{i}\right) \text {. }
$$

Theorem 4.1. Let $w_{i}=\exp \left(-Q_{i}\right) \in \mathcal{F}\left(C^{2}+\right), i=1,2, \cdots, s$, and let $1 \leq p \leq \infty$. Let a constant $\gamma \geq 0$ be fixed. We suppose that $g:=g\left(x_{1}, x_{2}, \cdots, x_{s}\right)$ is absolutely continuous on $\mathbb{R}^{s}$ and $W g^{(1,1, \cdots, 1)} \in L^{p}\left(\mathbb{R}^{s}\right)$. Then we have

$$
\begin{aligned}
& \left\|\prod_{i=1}^{s}\left(Q_{i}^{\prime} w_{i}\right) g\right\|_{L^{p}\left(\left|x_{i}\right| \leq \gamma, i=1, \cdots, s\right)} \\
& \leq C\left\{\int_{\left|x_{1}\right| \leq \gamma} \cdots \int_{\mid x_{s} \leq \gamma_{0}} \sum_{0 \leq j_{1} \leq 1}\left|w_{1, j_{1}}\left(x_{1}\right)\right|^{p} \cdots \sum_{0 \leq j_{s} \leq 1}\left|w_{s, j_{s}}\left(x_{s}\right)\right|^{p}\right. \\
& \left.\times\left|g^{\left(j_{1}, j_{2}, \cdots, j_{s}\right)}\left(y_{1}, y_{2}, \cdots, y_{s}\right)\right|^{p} \mathrm{~d} x_{s} \cdots \mathrm{d} x_{1}\right\}^{1 / p},
\end{aligned}
$$

where we set for each $j_{i}=0$ or $1, i=1,2, \cdots, s$,

$$
y_{i}= \begin{cases}\gamma, & j_{i}=0 \\ x_{i}, & j_{i}=1\end{cases}
$$

Furthermore, let $r$ be a positive integer, and let for each $i=1,2, \cdots, s$, $w_{i}=\exp \left(-Q_{i}\right) \in \mathcal{F}_{\lambda}\left(C^{3}+\right) \subset \mathcal{F}\left(C^{2}+\right) \quad(0<\lambda<3 / 2)$. We suppose that $g^{(r-1, r-1, \cdots, r-1)}$ is absolutely continuous and $W g^{(r, r, \cdots, r)} \in L^{p}\left(\mathbb{R}^{s}\right)$. Then we have

$$
\left\{\int_{\mathbb{R}^{s}}\left|w_{1, j_{1}}\left(x_{1}\right)\right|^{p}\left|w_{s, j_{s}}\left(x_{s}\right)\right|^{p}\left|g^{\left(j_{1}, j_{2}, \cdots, j_{s}\right)}\left(y_{1}, y_{2}, \cdots, y_{s}\right)\right|^{p} \mathrm{~d} x_{s} \cdots \mathrm{d} x_{1}\right\}^{1 / p}<\infty,
$$

for each $0 \leq j_{i} \leq r, i=1,2, \cdots, s$ with

$$
y_{i}= \begin{cases}\gamma, & 0 \leq j_{i} \leq r-1 \\ x_{i}, & j_{i}=r\end{cases}
$$

and

$$
\begin{aligned}
& \left\|\left(\prod_{i=1}^{s} Q_{i}^{\prime}\right)^{r} W g\right\|_{L^{p}\left(\left|x_{i}\right| \geq \gamma, i=1, \cdots, s\right)} \\
& \leq C\left\{\int_{\left|x_{1}\right| \geq \gamma} \cdots \int_{\left|x_{s}\right| \geq \gamma} \sum_{0 \leq j_{1} \leq r}\left|w_{1, j_{1}}\left(x_{1}\right)\right|^{p} \cdots \sum_{0 \leq j_{s} \leq r}\left|w_{s, j_{s}}\left(x_{s}\right)\right|^{p}\right. \\
& \left.\quad \times\left|g^{\left(j_{1}, j_{2}, \cdots, j_{s}\right)}\left(y_{1}, y_{2}, \cdots, y_{s}\right)\right|^{p} \mathrm{~d} x_{s} \cdots \mathrm{d} x_{1}\right\}^{1 / p}<\infty .
\end{aligned}
$$

Proposition 4.2 ([8], Theorem 9, cf. [9], Lemma 3.4.4). Let $w=\exp (-Q) \in \mathcal{F}\left(C^{2}+\right)$ and a constant $\gamma \geq 0$ be fixed.

(a) We have

$$
\left|Q^{\prime}(x) w(x) \int_{\gamma}^{x} w^{-1}(t) \mathrm{d} t\right| \leq C, \quad|x| \geq \gamma
$$


(b) Let $1 \leq p \leq \infty$, and let $r$ be a positive integer. If $g$ is absolutely continuous, $g(\gamma)=0$ and $w g^{\prime} \in L^{p}(\mathbb{R})$, then

$$
\left\|Q^{\prime} w g\right\|_{L^{p}(|x| \geq \gamma)} \leq C\left\|w g^{\prime}\right\|_{L^{p}(|x| \geq \gamma)} .
$$

When $w=\exp (-Q) \in \mathcal{F}_{\lambda}\left(C^{r+1}+\right) \subset \mathcal{F}\left(C^{2}+\right) \quad(0<\lambda<(r+1) / r)$, and $g^{(r-1)}$ is absolutely continuous, $g^{(j)}(\gamma)=0, j=0,1, \cdots, r-1$ with $w g^{(r)} \in L^{p}(\mathbb{R})$, we see

$$
\left\|\left(Q^{\prime}\right)^{r} w g\right\|_{L^{p}(|x|>\gamma)} \leq C\left\|w g^{(r)}\right\|_{L^{p}(|x| \geq \gamma)} \cdot
$$

Proposition 4.3 ([3], Theorem 4.2). Let $w=\exp (-Q) \in \mathcal{F}_{\lambda}\left(C^{3}+\right) \subset \mathcal{F}\left(C^{2}+\right)$. Then for $\alpha \in \mathbb{R}$, we can construct a new weight $w_{\alpha} \in \mathcal{F}\left(C^{2}+\right)$ such that

$$
\left(1+\left|Q^{\prime}(x)\right|\right)^{\alpha} w(x) \sim w_{\alpha}(x)=\exp \left(-Q_{\alpha}\right)
$$

on $\mathbb{R},(1 / c) a_{n}(w) \leq a_{n}\left(w_{\alpha}\right) \leq c a_{n}(w)$ ( $c$ is an absolutely constant) on $\mathbb{N}$ and $T_{w_{\alpha}}(x) \sim T_{w}(x)$ hold on $\mathbb{R}$. Furthermore, we see

$$
Q_{\alpha}^{(j)}(x) \sim Q^{(j)}(x)(j=0,1) \text { for }|x| \geq \gamma>0 .
$$

Proof of Theorem 4.1. For the proof of (4.1) we may put $r=1$ with $w_{i}=\exp \left(-Q_{i}\right) \in \mathcal{F}\left(C^{2}+\right), i=1,2, \cdots, s$ in the proof of (4.4) below. So we prove only (4.4). We use Proposition 4.2 and 4.3 repeatedly.

$$
\begin{aligned}
& \left\{\int_{\left\|x_{s}\right\| \geq \gamma} \cdots \int_{\left\|x_{1}\right\| \geq \gamma}\left|\prod_{i=1}^{s}\left(Q_{i}^{\prime r}\left(x_{i}\right) w_{i}\left(x_{i}\right)\right) g\left(x_{1}, \cdots, x_{s}\right)\right|^{p} \mathrm{~d} x_{1} \cdots \mathrm{d} x_{s}\right\}^{1 / p} \\
& =\left\{\int_{\left\|x_{s}\right\| \geq \gamma} \cdots \int_{\left\|x_{2}\right\| \geq \gamma}\left|\prod_{i=2}^{s}\left(Q_{i}^{\prime r}\left(x_{i}\right) w_{i}\left(x_{i}\right)\right)\right|^{p}\right. \\
& \left.\quad \times \int_{\left\|x_{1}\right\| \geq \gamma}\left|Q_{1}^{\prime r}\left(x_{1}\right) w_{1}\left(x_{1}\right) g\left(x_{1}, \cdots, x_{s}\right)\right|^{p} \mathrm{~d} x_{1} \cdots \mathrm{d} x_{s}\right\}^{1 / p} \\
& \leq C_{1,0}\left[\left\{\int_{\left\|x_{s}\right\| \geq \gamma} \cdots \int_{\left\|x_{2}\right\| \geq \gamma}\left|\prod_{i=2}^{s}\left(Q_{i}^{\prime r}\left(x_{i}\right) w_{i}\left(x_{i}\right)\right)\right|^{p}\right.\right. \\
& \left.\left.\quad \times \int_{\left\|x_{1}\right\| \geq \gamma}\left|Q_{1,1}^{\prime}\left(x_{1}\right) w_{1,1}\left(x_{1}\right) g\left(x_{1}, \cdots, x_{s}\right)\right|^{p} \mathrm{~d} x_{1} \cdots \mathrm{d} x_{s}\right\}^{1 / p}\right],
\end{aligned}
$$

where $\left(Q_{1}^{\prime}\right)^{r} w_{1}=Q_{1}^{\prime}\left(Q_{1}^{\prime}\right)^{r-1} w_{1} \sim Q_{1}^{\prime} w_{1,1} \sim Q_{1,1}^{\prime} w_{1,1}, w_{1,1}=\mathrm{e}^{-Q_{1,1}}$,

$$
\begin{aligned}
\leq & C_{1,0}\left[\left\{\int_{\left\|x_{s}\right\| \geq \gamma} \cdots \int_{\left\|x_{2}\right\| \geq \gamma}\left|\prod_{i=2}^{s}\left(Q_{i}^{\prime r}\left(x_{i}\right) w_{i}\left(x_{i}\right)\right)\right|^{p}\right.\right. \\
& \left.\times \int_{\left\|x_{1}\right\| \geq \gamma}\left|Q_{1,1}^{\prime}\left(x_{1}\right) w_{1,1}\left(x_{1}\right)\left(g\left(x_{1}, \cdots, x_{s}\right)-g\left(\gamma, x_{2}, \cdots, x_{s}\right)\right)\right|^{p} \mathrm{~d} x_{1} \cdots \mathrm{d} x_{s}\right\}^{1 / p} \\
& +\left\{\int_{\left\|x_{s}\right\| \geq \gamma} \cdots \int_{\left\|x_{2}\right\| \geq \gamma}\left|\prod_{i=2}^{s}\left(Q_{i}^{\prime r}\left(x_{i}\right) w_{i}\left(x_{i}\right)\right)\right|^{p}\right. \\
& \left.\left.\times \int_{\left\|x_{1}\right\| \geq \gamma}\left|Q_{1,1}^{\prime}\left(x_{1}\right) w_{1,1}\left(x_{1}\right) g\left(\gamma, x_{2}, \cdots, x_{s}\right)\right|^{p} \mathrm{~d} x_{1} \cdots \mathrm{d} x_{s}\right\}^{1 / p}\right] \\
\leq & C_{1,1}\left[\left\{\int_{\left\|x_{s}\right\| \geq \gamma} \cdots \int_{\left\|x_{2}\right\| \geq \gamma}\left|\prod_{i=2}^{s}\left(Q_{i}^{\prime r}\left(x_{i}\right) w_{i}\left(x_{i}\right)\right)\right|^{p}\right.\right. \\
& \left.\times \int_{\left\|x_{1}\right\| \geq \gamma}\left|w_{1,1}\left(x_{1}\right) g^{(1,0, \cdots, 0)}\left(x_{1}, \cdots, x_{s}\right)\right|^{p} \mathrm{~d} x_{1} \cdots \mathrm{d} x_{s}\right\}^{1 / p} \\
& \left.+\left\{\int_{\left\|x_{s}\right\| \geq \gamma} \cdots \int_{\left\|x_{1}\right\| \geq \gamma}\left|\prod_{i=1}^{s}\left(Q_{i}^{\prime r}\left(x_{i}\right) w_{i}\left(x_{i}\right)\right) g\left(\gamma, x_{2}, \cdots, x_{s}\right)\right|^{p} \mathrm{~d} x_{1} \cdots \mathrm{d} x_{s}\right\}^{1 / p}\right]
\end{aligned}
$$


by $Q_{1,1}^{\prime} w_{1,1} \sim\left(Q_{1}^{\prime}\right)^{r} w_{1}$,

$$
\begin{aligned}
& \leq C_{1,1}^{\prime}\left[\left\{\int_{\| x_{s} \mid \geq \gamma} \cdots \int_{\left\|x_{2}\right\| \geq \gamma}\left|\prod_{i=2}^{s}\left(Q_{i}^{\prime r}\left(x_{i}\right) w_{i}\left(x_{i}\right)\right)\right|^{p}\right.\right. \\
& \left.\times \int_{\left\|x_{1}\right\| \geq \gamma}\left|Q_{1,2}^{\prime}\left(x_{1}\right) w_{1,2}\left(x_{1}\right) g^{(1,0, \cdots, 0)}\left(x_{1}, \cdots, x_{s}\right)\right|^{p} \mathrm{~d} x_{1} \cdots \mathrm{d} x_{s}\right\}^{1 / p} \\
& \text { where } w_{1,1} \sim\left(Q_{1}^{\prime}\right)^{r-1} w \sim Q_{1,2}^{\prime} w_{1,2}, w_{1,2}=\mathrm{e}^{-Q_{1,2}} \text {, } \\
& \left.+\left\{\int_{\left\|x_{s}\right\| \geq \gamma} \cdots \int_{\| x_{1} \mid \geq \gamma}\left|\prod_{i=1}^{s}\left(Q_{i}^{\prime r}\left(x_{i}\right) w_{i}\left(x_{i}\right)\right) g\left(\gamma, x_{2}, \cdots, x_{s}\right)\right|^{p} \mathrm{~d} x_{1} \cdots \mathrm{d} x_{s}\right\}^{1 / p}\right] \\
& \leq C_{1,2}\left[\left\{\int_{\left\|x_{s}\right\| \geq \gamma} \cdots \int_{\left\|x_{2}\right\| \geq \gamma}\left|\prod_{i=2}^{s}\left(Q_{i}^{\prime r}\left(x_{i}\right) w_{i}\left(x_{i}\right)\right)\right|^{p}\right.\right. \\
& \left.\times \int_{\left\|x_{x}\right\| \geq \gamma}\left|w_{1,2}\left(x_{1}\right) g^{(2,0, \cdots, 0)}\left(x_{1}, \cdots, x_{s}\right)\right|^{p} \mathrm{~d} x_{1} \cdots \mathrm{d} x_{s}\right\}^{1 / p} \\
& +\left\{\int_{\left\|x_{s}\right\| \geq \gamma} \cdots \int_{\left\|x_{x}\right\| \geq \gamma}\left|\prod_{i=2}^{s}\left(Q_{i}^{\prime r}\left(x_{i}\right) w_{i}\left(x_{i}\right)\right)\right|^{p}\right. \\
& \left.\times\left|Q_{1}^{\prime r-1}\left(x_{1}\right) w_{1}\left(x_{1}\right) g^{(1,0, \cdots, 0)}\left(\gamma, x_{2}, \cdots, x_{s}\right)\right|^{p} \mathrm{~d} x_{1} \cdots \mathrm{d} x_{s}\right\}^{1 / p} \\
& \left.+\left\{\int_{\left\|x_{s}\right\| \geq \gamma} \cdots \int_{\left\|x_{1}\right\| \geq \gamma}\left|\prod_{i=1}^{s}\left(Q_{i}^{\prime r}\left(x_{i}\right) w_{i}\left(x_{i}\right)\right) g\left(\gamma, x_{2}, \cdots, x_{s}\right)\right|^{p} \mathrm{~d} x_{1} \cdots \mathrm{d} x_{s}\right\}^{1 / p}\right] \\
& \leq \cdots \\
& \leq C_{1, r}\left[\left\{\int_{\left\|x_{s}\right\| \geq \gamma} \cdots \int_{\left\|x_{2}\right\| \geq \gamma}\left|\prod_{i=2}^{s}\left(Q_{i}^{\prime r}\left(x_{i}\right) w_{i}\left(x_{i}\right)\right)\right|^{p}\right.\right. \\
& \left.\times \int_{\left\|x_{1}\right\| \geq \gamma}\left|w_{1}\left(x_{1}\right) g^{(r, 0, \cdots, 0)}\left(x_{1}, \cdots, x_{s}\right)\right|^{p} \mathrm{~d} x_{1} \cdots \mathrm{d} x_{s}\right\}^{1 / p} \\
& +\left\{\int_{\left\|x_{s}\right\| \geq \gamma} \cdots \int_{\left\|x_{x}\right\| \geq \gamma}\left|\prod_{i=2}^{s}\left(Q_{i}^{\prime r}\left(x_{i}\right) w_{i}\left(x_{i}\right)\right)\right|^{p}\right. \\
& \left.\times\left|Q_{1}^{\prime}\left(x_{1}\right) w_{1}\left(x_{1}\right) g^{(1,0, \cdots, 0)}\left(\gamma, x_{2}, \cdots, x_{s}\right)\right|^{p} \mathrm{~d} x_{1} \cdots \mathrm{d} x_{s}\right\}^{1 / p} \\
& +\cdots \\
& +\left\{\int_{\left\|x_{s}\right\| \geq \gamma} \ldots \int_{\left\|x_{1}\right\| \geq \gamma}\left|\prod_{i=2}^{s}\left(Q_{i}^{\prime r}\left(x_{i}\right) w_{i}\left(x_{i}\right)\right)\right|^{p}\right. \\
& \left.\times\left|Q_{1}^{r-1}\left(x_{1}\right) w_{1}\left(x_{1}\right) g^{(1,0, \cdots, 0)}\left(\gamma, x_{2}, \cdots, x_{s}\right)\right|^{p} \mathrm{~d} x_{1} \cdots \mathrm{d} x_{s}\right\}^{1 / p} \\
& \left.+\left\{\int_{\left\|x_{s}\right\| \geq \gamma} \cdots \int_{\left\|x_{1}\right\| \geq \gamma}\left|\prod_{i=1}^{s}\left(Q_{i}^{\prime r}\left(x_{i}\right) w_{i}\left(x_{i}\right)\right) g\left(\gamma, x_{2}, \cdots, x_{s}\right)\right|^{p} \mathrm{~d} x_{1} \cdots \mathrm{d} x_{s}\right\}^{1 / p}\right] \\
& =C_{1, r}\left\{\int_{\left\|x_{s}\right\| \geq \gamma} \cdots \int_{\left\|x_{2}\right\| \geq \gamma}\left|\prod_{i=2}^{s}\left(Q_{i}^{\prime r}\left(x_{i}\right) w_{i}\left(x_{i}\right)\right)\right|^{p}\right. \\
& \left.\times\left.\int_{\left\|x_{1}\right\| \geq \gamma_{0 \leq j_{1} \leq r}} \sum_{1, j_{1}}\left(x_{1}\right) g^{\left(j_{1}, 0, \cdots, 0\right)}\left(y_{1}, x_{2}, \cdots, x_{s}\right)\right|^{p} \mathrm{~d} x_{1} \cdots \mathrm{d} x_{s}\right\}^{1 / p},
\end{aligned}
$$

where

$$
y_{1}= \begin{cases}\gamma, & 0 \leq j_{1} \leq r-1 \\ x_{1}, & j_{1}=r\end{cases}
$$


We continue this manner with respect to $x_{2}, x_{3}, \cdots, x_{s}$. Then we can easily obtain as follows:

$$
\begin{aligned}
& \left\{\int_{\left|x_{s}\right| \geq \gamma} \cdots \int_{\left|x_{1}\right| \geq \gamma}\left|\prod_{i=1}^{s}\left(Q_{i}^{\prime r}\left(x_{i}\right) w_{i}\left(x_{i}\right)\right) g\left(x_{1}, \cdots, x_{s}\right)\right|^{p} \mathrm{~d} x_{1} \cdots \mathrm{d} x_{s}\right\}^{1 / p} \\
& \leq C_{1, r}\left\{\int_{\left|x_{s}\right| \geq \gamma} \cdots \int_{\left|x_{2}\right|>\gamma}\left|\prod_{i=2}^{s}\left(Q_{i}^{\prime r}\left(x_{i}\right) w_{i}\left(x_{i}\right)\right)\right|^{p}\right. \\
& \left.\times\left.\int_{\left|x_{1}\right| \geq \gamma_{0 \leq j_{1} \leq r}} \sum_{1, j_{1}}\left(x_{1}\right) g^{\left(j_{1}, 0, \cdots, 0\right)}\left(y_{1}, x_{2}, \cdots, x_{s}\right)\right|^{p} \mathrm{~d} x_{1} \cdots \mathrm{d} x_{s}\right\}^{1 / p} \\
& \leq C_{2}\left\{\int_{\left|x_{1}\right| \geq \gamma} \sum_{0 \leq j_{1} \leq r}\left|w_{1, j_{1}}\left(x_{1}\right)\right|^{p} \int_{\left|x_{s}\right| \geq \gamma} \cdots \int_{\left|x_{3}\right| \geq \gamma}\left|\prod_{i=3}^{s}\left(Q_{i}^{\prime r}\left(x_{i}\right) w_{i}\left(x_{i}\right)\right)\right|^{p}\right. \\
& \left.\left.\times \int_{\left|x_{2}\right| \geq \gamma_{0 \leq j_{2} \leq r}} \sum_{2, j_{2}} \mid w_{2}\right)\left.g^{\left(j_{1}, j_{2}, 0, \cdots, 0\right)}\left(y_{1}, y_{2}, x_{3}, \cdots, x_{s}\right)\right|^{p} \mathrm{~d} x_{2} \cdots \mathrm{d} x_{s} \mathrm{~d} x_{1}\right\}^{1 / p} \\
& =C_{2}\left\{\int_{\left|x_{1}\right| \geq \gamma} \int_{\left|x_{2}\right| \geq \gamma} \sum_{0 \leq j_{1} \leq r}\left|w_{1, j_{1}}\left(x_{1}\right)\right|^{p} \sum_{0 \leq j_{2} \leq r}\left|w_{2, j_{2}}\left(x_{2}\right)\right|^{p} \int_{\left|x_{s}\right| \geq \gamma} \cdots \int_{\left|x_{4}\right| \geq \gamma}\left|\prod_{i=4}^{s}\left(Q_{i}^{\prime r}\left(x_{i}\right) w_{i}\left(x_{i}\right)\right)\right|^{p}\right. \\
& \left.\times \int_{\left|x_{3}\right| \geq \gamma}\left|w_{3, j_{3}}\left(x_{3}\right) g^{\left(j_{1}, j_{2}, j_{3}, 0, \cdots, 0\right)}\left(y_{1}, y_{2}, y_{3}, x_{4}, \cdots, x_{s}\right)\right|^{p} \times \mathrm{d} x_{3} \cdots \mathrm{d} x_{s} \mathrm{~d} x_{2} \mathrm{~d} x_{1}\right\}^{1 / p} \\
& \leq \ldots \\
& \leq C_{r}\left\{\int_{\left|x_{1}\right| \geq \gamma} \cdots \int_{\left|x_{s}\right| \geq \gamma} \sum_{0 \leq j_{1} \leq r}\left|w_{1, j_{1}}\left(x_{1}\right)\right|^{p} \cdots \sum_{0 \leq j_{s} \leq r}\left|w_{s, j_{s}}\left(x_{s}\right)\right|^{p}\right. \\
& \left.\times\left. g^{\left(j_{1}, j_{2}, \cdots, j_{s}\right)}\left(y_{1}, y_{2}, \cdots, y_{s}\right)\right|^{p} \mathrm{~d} x_{s} \cdots \mathrm{d} x_{1}\right\}^{1 / p} \text {, }
\end{aligned}
$$

where for each $0 \leq j_{i} \leq r, i=1,2, \cdots, s$. We set (4.3).

Let $W g^{(r, \cdots, r)} \in L^{p}\left(\mathbb{R}^{s}\right)$. Then we need to show

$$
\begin{aligned}
A:= & \left\{\int_{\left|x_{1}\right| \geq \gamma} \cdots \int_{\left|x_{s}\right| \geq \gamma} \sum_{0 \leq j_{1} \leq r}\left|w_{1, j_{1}}\left(x_{1}\right)\right|^{p} \cdots \sum_{0 \leq j_{s} \leq r}\left|w_{s, j_{s}}\left(x_{s}\right)\right|^{p}\right. \\
& \left.\times\left|g^{\left(j_{1}, j_{2}, \cdots, j_{s}\right)}\left(y_{1}, y_{2}, \cdots, y_{s}\right)\right|^{p} \mathrm{~d} x_{s} \cdots \mathrm{d} x_{1}\right\}^{1 / p}<\infty .
\end{aligned}
$$

We rearrange $\left(x_{1}, x_{2}, \cdots, x_{s}\right)$ as $x_{k_{i}}=y_{i}=x_{i}, t+1 \leq k_{i} \leq s$ if $j_{i}=r$, and as $x_{k_{i}}=y_{i}=\gamma<1 \leq k_{i} \leq t$ if $0 \leq j_{i} \leq r-1$, where $0 \leq t \leq s$. Then we set $f\left(x_{k_{1}}, x_{k_{2}}, \cdots, x_{k_{s}}\right):=g\left(x_{1}, x_{2}, \cdots, x_{s}\right)$. We see

$$
g^{\left(j_{1}, j_{2}, \cdots, j_{s}\right)}\left(y_{1}, y_{2}, \cdots, y_{s}\right)=f^{\left(j_{k_{1}}, j_{k_{2}}, \cdots, j_{k_{s}}\right)}\left(\gamma, \cdots, \gamma, x_{t+1}, x_{t+2}, \cdots, x_{s}\right) .
$$

Then we have

$$
\begin{aligned}
A & =\left\|\prod_{i=1}^{t} w_{k_{i}}\right\| \prod_{i=t+1}^{s} w_{k_{i}} f^{\left(j_{k_{1}}, \cdots, j_{k_{s}}\right)}\left(\gamma, \cdots, \gamma, x_{t+1}, \cdots, x_{s}\right)\left\|_{L^{p}\left(\mathbb{R}_{t+1 \leq}^{s-t}\right)}\right\|_{L^{p}\left(\mathbb{R}_{s t}^{t}\right)} \\
& =:\left\|\prod_{i=1}^{t} w_{k_{i}} \mid h^{\left(j_{k_{1}}, \cdots, j_{k_{t}}\right)}(\gamma, \cdots, \gamma)\right\| \|_{L^{p}\left(\mathbb{R}_{s t}^{t}\right)} \\
& \leq C\left|h^{\left(j_{k_{1}}, \cdots, j_{k_{t}}\right)}(\gamma, \cdots, \gamma)\right|\left\|\prod_{i=1}^{t} w_{k_{i}}\right\|_{L^{p}\left(\mathbb{R}_{s t}^{t}\right)}<\infty .
\end{aligned}
$$


We can generalize Theorem 4.1 easily. We give a class of nonnegative integers $\left(j_{1}, j_{2}, \cdots, j_{s}\right)$, and set $J_{s}:=\left(j_{1}, j_{2}, \cdots, j_{s}\right)$. For $r_{i} \geq 1, i=1,2, \cdots, s$ we set $R_{s}:=\left(r_{1}, r_{2}, \cdots, r_{s}\right)$. Then we consider the order as follows:

$$
K_{s}:=\left(k_{1}, k_{2}, \cdots, k_{s}\right) \leq R_{s}:=\left(r_{1}, r_{2}, \cdots, r_{s}\right)
$$

means

$$
k_{i} \leq r_{i}(i=1,2, \cdots, s) .
$$

Corollary 4.4. Let $K_{s}=\left(k_{1}, k_{2}, \cdots, k_{s}\right) \leq R_{s}=\left(r_{1}, r_{2}, \cdots, r_{s}\right)$ be classes of nonnegative integers, where $r_{i} \geq 1, i=1,2, \cdots, s$. For each $i=1,2, \cdots, s$, we suppose $w_{i}=\exp \left(-Q_{i}\right) \in \mathcal{F}_{\lambda}\left(C^{r+1}+\right) \subset \mathcal{F}\left(C^{2}+\right) \quad(0<\lambda<(r+1) / r)$. If $g^{\left(r_{1}-1, r_{2}-1, \cdots, r_{s}-1\right)}$ is absolutely continuous, and $W^{\left(r_{1}, r_{2}, \cdots, r_{s}\right)} \in L^{p}\left(\mathbb{R}^{s}\right)$, then we see

$$
\begin{aligned}
& \left\|\left(\prod_{i=1}^{s} Q_{i}^{\prime}\right)^{r_{i}-k_{i}} W g^{\left(k_{1}, \cdots, k_{s}\right)}\right\|_{L^{p}\left(\left|x_{i}\right| \gamma \gamma, i=1, \cdots, s\right)} \\
& \leq C\left\{\int_{\left|x_{1}\right| \geq \gamma} \cdots \int_{\left|x_{s}\right| \geq \gamma_{k_{1} \leq j_{1} \leq r_{1}}}\left|w_{1, j_{1}-k_{1}}\left(x_{1}\right)\right|^{p} \cdots \sum_{k_{s} \leq j_{s} \leq r_{s}}\left|w_{s, j_{s}-k_{s}}\left(x_{s}\right)\right|^{p}\right. \\
& \left.\times\left|g^{\left(j_{1}, j_{2}, \cdots, j_{s}\right)}\left(y_{1}, y_{2}, \cdots, y_{s}\right)\right|^{p} \mathrm{~d} x_{s} \cdots \mathrm{d} x_{1}\right\}^{1 / p}<\infty,
\end{aligned}
$$

where for each $i=1,2, \cdots, s$ we set

$$
y_{i}= \begin{cases}\gamma, & k_{i} \leq j_{i} \leq r_{i}-1 \\ x_{i}, & j_{i}=r_{i}\end{cases}
$$

We remark that $W g^{\left(r_{1}, \cdots, r_{s}\right)} \in L^{p}\left(\mathbb{R}^{s}\right)$ means $W g^{\left(k_{1}, \cdots, k_{s}\right)} \in L^{p}\left(\mathbb{R}^{s}\right)$ for $0 \leq K_{s}=\left(k_{1}, \cdots, k_{s}\right) \leq R_{s}=\left(r_{1}, \cdots, r_{s}\right)$.

\section{Degree of Approximation}

We define the degree of approximation for $W f \in L^{p}\left(\mathbb{R}^{s}\right)$ as follows:

$$
E_{n, p ; s}(W, f):=\inf _{P \in \mathcal{P}_{n ; s}\left(\mathbb{R}^{s}\right)}\|W(f-P)\|_{L^{p}\left(\mathbb{R}^{s}\right)} .
$$

Using this $E_{n, p ; s}(W, f)$, we can estimate the degree of approximation of $W f \in L^{p}\left(\mathbb{R}^{s}\right)$ from $\mathcal{P}_{n ; s}\left(\mathbb{R}^{s}\right)$.

Theorem 5.1. (1) Let $w_{i} \in \mathcal{F}\left(C^{2}+\right)(i=1,2, \cdots, s)$ and let $1 \leq p \leq \infty, W f \in L^{p}\left(\mathbb{R}^{s}\right)$. Furthermore, we suppose (3.3). Then we have

$$
\left\|\frac{W}{\prod_{i=1}^{s} T_{i}\left(x_{i}\right)^{1 / 4}}\left(f-v_{n}^{[s]}(f)\right)\right\|_{L^{p}\left(\mathbb{R}^{s}\right)} \leq C E_{n, p ; s}(W, f) .
$$

(2) If $w_{i} \in \mathcal{F}_{\lambda}\left(C^{3}+\right)(i=1,2, \cdots, s), 0<\lambda<3 / 2$, and let $\left\|\left(\prod_{i=1}^{s} T_{i}^{1 / 4}\right) W f\right\|_{L^{p}\left(\mathbb{R}^{s}\right)}<\infty$, then we have

$$
\left\|W\left(f-v_{n}^{[s]}(f)\right)\right\|_{L^{p}\left(\mathbb{R}^{s}\right)} \leq C E_{n, p ; s}\left(\left(\prod_{i=1}^{s} T_{i}^{1 / 4}\right) W, f\right) .
$$


(3) Let $w_{i} \in \mathcal{F}\left(C^{2}+\right)(i=1,2, \cdots, s)$ and let $1 \leq p \leq \infty, W f \in L^{p}\left(\mathbb{R}^{s}\right)$. Then we have

$$
\left\|W\left(f-v_{n}^{[s]}(f)\right)\right\|_{L^{p}\left(\mathbb{R}^{s}\right)} \leq C\left(\prod_{i=1}^{s} T_{i}^{1 / 4}\left(a_{n}^{(i)}\right)\right) E_{n, p ; s}(W, f) .
$$

(4) Furthermore, let $w_{i} \in \mathcal{F}_{\lambda}\left(C^{3}+\right)(0<\lambda<3 / 2), i=1,2, \cdots, s \quad$. If $f \in L_{W^{\delta}}^{p^{*}}\left(\mathbb{R}^{s}\right)$ for some $0<\delta<1$, then we have

$$
E_{n, p ; s}(W, f) \rightarrow 0 \text { as } n \rightarrow \infty \text {. }
$$

Proof. (1) There exists $P \in \mathcal{P}_{n}$ such that $\|W(f-P)\|_{L^{p}\left(\mathbb{R}^{s}\right)} \leq C E_{n, p ; s}(W, f)$. Therefore, by Lemma 3.9 (1)

$$
\begin{aligned}
& \left\|\frac{W}{\prod_{i=1}^{s} T_{i}^{1 / 4}}\left(f-v_{n}^{[s]}(f)\right)\right\|_{L^{p}\left(\mathbb{R}^{s}\right)} \\
& =\left\|\frac{W}{\prod_{i=1}^{s} T_{i}^{1 / 4}}(f-P)\right\|_{L^{p}\left(\mathbb{R}^{s}\right)}+\left\|\frac{W}{\prod_{i=1}^{s} T_{i}^{1 / 4}} v_{n}^{[s]}(f-P)\right\|_{L^{p}\left(\mathbb{R}^{s}\right)} \\
& \leq C\|W(f-P)\|_{L^{p}\left(\mathbb{R}^{s}\right)} \leq C E_{n, p ; s}(W, f) .
\end{aligned}
$$

(2) We see $W(X) \prod_{i=1}^{s} T_{i}^{1 / 4}\left(x_{i}\right) \sim \tilde{W}(X)=\prod_{i=1}^{s} \tilde{w}_{i}\left(x_{i}\right)$, $T_{i}^{1 / 4} w \sim \tilde{W}_{i} \in \mathcal{F}\left(C^{2}+\right), i=1,2, \cdots, s$. Then, there exists $P \in \mathcal{P}_{n}$ such that $\|\tilde{W}(f-P)\|_{L^{p}\left(\mathbb{R}^{s}\right)} \leq C E_{n-1, p ; s}(\tilde{W}, f)$. Therefore, by Lemma 3.9 (2)

$$
\begin{aligned}
& \left\|W\left(f-v_{n}^{[s]}(f)\right)\right\|_{L^{p}\left(\mathbb{R}^{s}\right)} \\
& =\|W(f-P)\|_{L^{p}\left(\mathbb{R}^{s}\right)}+\left\|W v_{n}^{[s]}(f-P)\right\|_{L^{p}\left(\mathbb{R}^{s}\right)} \\
& \leq C E_{n, p ; s}(\tilde{W}, f) \leq C E_{n, p ; s}\left(\left(\prod_{i=1}^{s} T_{i}^{1 / 4}\right) W, f\right) .
\end{aligned}
$$

(3) Similarly, we have (3).

(4) It follows from Theorem 3.3. \#

Theorem 5.2. Let $w_{i} \in \mathcal{F}_{\lambda}\left(C^{3}+\right)(i=1,2, \cdots, s), 0<\lambda<3 / 2$, and let $1 \leq p \leq \infty$. Then if $W f \in L_{p}\left(\mathbb{R}^{s}\right)$, we have

$$
\left\|\frac{W}{\prod_{i=1}^{s} T_{i}^{\left(2 j_{i}+1\right) / 4}} v_{n}^{[s]}(f)^{\left(j_{1}, \cdots, j_{s}\right)}\right\|_{L^{p}\left(\mathbb{R}^{s}\right)} \leq C \prod_{i=1}^{s}\left(\frac{n}{a_{n}^{(i)}}\right)^{j_{i}}\|W f\|_{L^{p}\left(\mathbb{R}^{s}\right)},
$$

and

$$
\left\|W v_{n}^{[s]}(f)^{\left(j_{1}, \cdots, j_{s}\right)}\right\|_{L^{p}\left(\mathbb{R}^{s}\right)} \leq C \prod_{i=1}^{s}\left(\frac{n}{a_{n}^{(i)}}\right)^{j_{i}}\left(\prod_{i=1}^{s} T_{i}^{\left(2 j_{i}+1\right) / 4}\left(a_{n}^{(i)}\right)\right)\|W f\|_{L^{p}\left(\mathbb{R}^{s}\right)} .
$$

Proposition 5.3 ([10], Lemma 2.5, [2], Corollary 10.2). Let $1 \leq p \leq \infty$ and $w \in \mathcal{F}_{\lambda}\left(C^{3}+\right)(0<\lambda<3 / 2)$. Then there exists a constant $C_{1}=C_{1}(w, p)>0$ such that, if $P \in \mathcal{P}_{n}(\mathbb{R}) \quad(n \in \mathbb{N})$, 


$$
\left\|\frac{w}{T^{j / 2}} P^{(j)}\right\|_{L^{p}\left(\mathbb{R}^{s}\right)} \leq C_{1}\left(\frac{n}{a_{n}}\right)^{j}\|w P\|_{L^{p}\left(\mathbb{R}^{s}\right)}, j \in \mathbb{N},
$$

and

$$
\left\|w P^{(j)}\right\|_{L^{p}\left(\mathbb{R}^{s}\right)} \leq C_{1}\left(\frac{n T\left(a_{n}\right)^{1 / 2}}{a_{n}}\right)^{j}\|w P\|_{L^{p}\left(\mathbb{R}^{s}\right)}, j \in \mathbb{N} .
$$

Proof of Theorem 5.2. We use Proposition 5.3 and Lemma 3.7 (1).

$$
\begin{aligned}
& \left\|\frac{W}{\prod_{i=1}^{s} T_{i}^{\left(2 j_{i}+1\right) / 4}} v_{n}^{[s]}(f)^{\left(j_{1}, \cdots, j_{s}\right)}\right\|_{\left.L^{p} \mathbb{R}^{s}\right)} \\
& \leq C \prod_{i=1}^{s}\left(\frac{n}{a_{n}^{(i)}}\right)^{j_{i}}\left\|\frac{W}{\prod_{i=1}^{s} T_{i}^{-1 / 4}} v_{n}^{[s]}(f)\right\|_{L^{p}\left(\mathbb{R}^{s}\right)} \\
& \leq C \prod_{i=1}^{s}\left(\frac{n}{a_{n}^{(i)}}\right)^{j_{i}}\|W f\|_{L^{p}\left(\mathbb{R}^{s}\right)},
\end{aligned}
$$

and further, using Theorem $A_{1}$ (the Markov-Bernstein inequality) in Appendix,

$$
\begin{aligned}
& \left\|W v_{n}^{[s]}(f)^{\left(j_{1}, \cdots, j_{s}\right)}\right\|_{L^{p}\left(\mathbb{R}^{s}\right)} \leq C \prod_{i=1}^{s}\left(\frac{n T_{i}^{1 / 2}\left(a_{n}^{(i)}\right)}{a_{n}^{(i)}}\right)^{j_{i}}\left\|W v_{n}^{[s]}(f)\right\|_{L^{p}\left(\mathbb{R}^{s}\right)} \\
& \leq C \prod_{i=1}^{s}\left(\frac{n T_{i}^{1 / 2}\left(a_{n}^{(i)}\right)}{a_{n}^{(i)}}\right)^{j_{i}}\left\|W v_{n}^{[s]}(f)\right\|_{L^{p}\left(\left|x_{i}\right| \leq a_{n}^{(i)}, i=1,2, \cdots, s\right)} \\
& \leq C \prod_{i=1}^{s}\left(\frac{n}{a_{n}^{(i)}}\right)^{j_{i}} \prod_{i=1}^{s} T_{i}^{\left(2 j_{i}+1\right) / 4}\left(a_{n}^{(i)}\right)\left\|\frac{W}{\prod_{i=1}^{s} T_{i}^{1 / 4}} v_{n}^{[s]}(f)\right\|_{L^{p}\left(\mathbb{R}^{s}\right)} \\
& \leq C \prod_{i=1}^{s}\left(\frac{n}{a_{n}^{(i)}}\right)^{j_{i}} \prod_{i=1}^{s} T_{i}^{\left(2 j_{i}+1\right) / 4}\left(a_{n}^{(i)}\right)\|W f\|_{L^{p}\left(\mathbb{R}^{s}\right)} .
\end{aligned}
$$

In the rest of only this section, we suppose

$$
w=\exp (-Q)=w_{i}=\exp \left(-Q_{i}\right), \quad i=1,2, \cdots, s,
$$

so

$$
a_{n}=a_{n}^{(i)}, T=T_{i}, \quad i=1,2, \cdots, s
$$

Let

$$
W_{i}:=W_{i}\left(x_{1}, \cdots, x_{i-1}, x_{i+1}, \cdots, x_{s}\right):=\prod_{j \neq i, 1 \leq j \leq s} w_{j}\left(x_{j}\right), \quad i=1,2, \cdots, s .
$$

In ([7], Corollary 8) we give the Favard-type inequalities:

Proposition 5.4 [7]. Let $w \in \mathcal{F}\left(C^{2}+\right)$, and let $r \geq 0$ be an integer. Let $1 \leq p \leq \infty$, and let $w f^{(r)} \in L_{p}(\mathbb{R})$. Then we have 


$$
E_{p, n}(f, w) \leq C\left(\frac{a_{n}}{n}\right)^{k}\left\|w f^{(k)}\right\|_{L_{p}(\mathbb{R})}, k=1,2, \cdots, r
$$

and equivalently,

$$
E_{p, n}(f, w) \leq C\left(\frac{a_{n}}{n}\right)^{k} E_{p, n-k}\left(f^{(k)}, w\right) .
$$

The following theorem is a generalization of Proposition 5.4.

Theorem 5.5. We suppose

$w_{j}=\exp \left(-Q_{j}\right) \in \mathcal{F}_{\lambda}\left(C^{3}+\right)(0<\lambda<3 / 2), j=1,2, \cdots, s$, and let (3.3) satisfy, that is,

$$
T_{j}\left(a_{n}\right) \leq c\left(\frac{n}{a_{n}}\right)^{2 / 3}, j=1,2, \cdots, s .
$$

Let $W f^{(r, r, \cdots, r)} \in L_{p}\left(\mathbb{R}^{s}\right)$ for some positive integer $r$. Then we have

$$
E_{n, p ; s}(W ; f) \leq C\left(\frac{a_{n}}{n}\right)^{r}\left\|T^{\langle s\rangle} W f^{(r, r, \cdots, r)}\right\|_{L^{p}\left(\mathbb{R}^{s}\right)} .
$$

Equivalently,

$$
E_{n, p ; s}(W ; f) \leq C\left(\frac{a_{n}}{n}\right)^{r} E_{n-r, p ; s}\left(T^{\langle s\rangle} W ; f^{(r, r, \cdots, r)}\right) .
$$

Proof. Using

$f-v_{n}^{[s]}=\left(f-v_{n}^{[1]}(f)\right)+\left(v_{n}^{[1]}(f)-v_{n}^{[2]}(f)\right)+\cdots+\left(v_{n}^{[s-1]}(f)-v_{n}^{[s]}(f)\right), \quad$ we get from Lemma 3.9 (2) and (3.2),

$$
\begin{aligned}
E_{n, p ; s} & \leq\left\|W\left(f-v_{n}^{[s]}(f)\right)\right\|_{L^{p}\left(\mathbb{R}^{s}\right)} \\
& \leq\left\|W\left(f-v_{n}^{[1]}(f)\right)\right\|_{L^{p}\left(\mathbb{R}^{s}\right)}+\sum_{j=2}^{s}\left\|W\left(v_{n}^{[j-1]}(f)-v_{n}^{[j]}(f)\right)\right\|_{L^{p}\left(\mathbb{R}^{s}\right)} \\
& \leq C\left[\left\|W\left(f-v_{n, 1}(f)\right)\right\|_{L^{p}\left(\mathbb{R}^{s}\right)}+\sum_{j=2}^{s}\left\|W v_{n}^{[j-1]}\left(f-v_{n, j}(f)\right)\right\|_{\left.L^{p}\left(\mathbb{R}^{s}\right)\right]}\right] \\
& \leq C\left[\left\|W\left(f-v_{n, 1}(f)\right)\right\|_{L^{p}\left(\mathbb{R}^{s}\right)}+\sum_{j=2}^{s}\left\|W\left(\prod_{k=1}^{j-1} T_{i}^{1 / 4}\right)\left(f-v_{n, j}(f)\right)\right\|_{L^{p}\left(\mathbb{R}^{s}\right)}\right]
\end{aligned}
$$

We estimate each term. From Proposition 5.4 with the weight $T_{j}^{1 / 4} w_{j}$,

$$
\begin{aligned}
& \left\|W\left(\prod_{k=1}^{j-1} T_{k}^{1 / 4}\right)\left(f-v_{n, j}(f)\right)\right\|_{L^{p}\left(\mathbb{R}^{s}\right)} \\
& =\left\|W_{i}\left(\prod_{k=1}^{j-1} T_{k}^{1 / 4}\right) w_{j}\left(f_{\hat{X}_{j}}-v_{n, j}\left(f_{\hat{X}_{j}}\right)\right)\right\|_{L^{p}\left(\mathbb{R}_{(j)}\right)} \|_{L^{p}\left(\mathbb{R}_{j}^{s-1}\right)} \\
& \leq C\left\|W_{i}\left(\prod_{k=1}^{j-1} T_{k}^{1 / 4}\right) E_{n, p}\left(T_{j}^{1 / 4} w_{j}, f_{\hat{X}_{j}}\right)\right\|_{L^{p}\left(\mathbb{R}_{j}^{s-1}\right)} \\
& \leq C\left(\frac{a_{n}}{n}\right)^{r}\left\|\prod_{i \neq j} w_{i}\left(\prod_{k=1}^{j-1} T_{k}^{1 / 4}\right)\right\| T_{j}^{1 / 4} w_{j} f_{\hat{X}_{j}}^{(r, 0, \cdots, 0)}\left\|_{L^{p}\left(\mathbb{R}_{(j)}\right)}\right\|_{L^{p}\left(\mathbb{R}_{j}^{s-1}\right)} .
\end{aligned}
$$


Now, we use Theorem 4.1 and the fact

$$
T_{i}\left(x_{i}\right) \leq Q_{i}^{\prime}\left(x_{i}\right)^{r}, i=1,2, \cdots, s \text {, }
$$

then we have

$$
\begin{aligned}
& \left\|W\left(\prod_{k=1}^{j-1} T_{k}^{1 / 4}\right)\left(f-v_{n, j}(f)\right)\right\|_{L^{p}\left(\mathbb{R}^{s}\right)} \\
& \leq C\left(\frac{a_{n}}{n}\right)^{r}\left\|\prod_{i \neq j} Q_{i}^{\prime}\left(x_{i}\right)^{r} w_{i}\right\| T_{j}^{1 / 4} w_{j} f_{\hat{X}_{j}}^{(r, 0, \cdots, 0)}\left\|_{L^{p}\left(\mathbb{R}_{(j)}\right)}\right\|_{L^{p}\left(\mathbb{R}_{j}^{s-1}\right)} \\
& \leq C\left(\frac{a_{n}}{n}\right)^{r}\left\|T^{\langle s\rangle} W f^{(r, r, \cdots, r)}\right\|_{L^{p}\left(\mathbb{R}^{s}\right)} .
\end{aligned}
$$

Consequently, we have

$$
\left\|W\left(f-v_{n}^{[s]}(f)\right)\right\|_{L^{p}\left(\mathbb{R}^{s}\right)} \leq C\left(\frac{a_{n}}{n}\right)^{r}\left\|T^{\langle s\rangle} W f^{(r, r, \cdots, r)}\right\|_{L^{p}\left(\mathbb{R}^{s}\right)} .
$$

Corollary 5.6. Under the conditions of Theorem 5.5, if $w$ is a Freud-type weight, then

$$
E_{n, p ; s}(W ; f) \leq C\left(\frac{a_{n}}{n}\right)^{r}\left\|W f^{(r, r, \cdots, r)}\right\|_{L^{p}\left(\mathbb{R}^{s}\right)} .
$$

Equivalently,

$$
E_{n, p ; s}(W ; f) \leq C\left(\frac{a_{n}}{n}\right)^{r} E_{n-r, p ; s}\left(W ; f^{(r, r, \cdots, r)}\right) .
$$

Let $1 \leq p \leq \infty$. For $\|W f\|_{L_{p}\left(\mathbb{R}^{s}\right)}<\infty$ we define the $\mathrm{K}$-functional $\mathcal{K}_{r, p}(W ; f, \delta)$ by

$$
K_{r, p ; s}(W ; f, \delta):=\inf _{g}\left\{\|W(f-g)\|_{L_{p}\left(\mathbb{R}^{s}\right)}+\delta^{r}\left\|W g^{(r, r, \cdots, r)}\right\|_{L_{p}\left(\mathbb{R}^{s}\right)}\right\},
$$

where the infimum is over all functions $g^{(r-1, r-1, \cdots, r-1)}$ which are absolutely continuous and $\left\|W g^{(r, r, \cdots, r)}\right\|_{L_{p}\left(\mathbb{R}^{s}\right)}<\infty$. We have the following.

Theorem 5.7. We suppose

$w_{j}=\exp \left(-Q_{j}\right) \in \mathcal{F}_{\lambda}\left(C^{3}+\right)(0<\lambda<3 / 2), j=1,2, \cdots, s$, and let

$$
T_{j}\left(a_{n}\right) \leq c\left(\frac{n}{a_{n}}\right)^{2 / 3}, j=1,2, \cdots, s .
$$

Let $1 \leq p \leq \infty$, and let $\left\|T^{\langle s\rangle} W f\right\|_{L_{p}\left(\mathbb{R}^{s}\right)}<\infty$. Then we have

$$
E_{n, p ; s}(W, f) \leq K_{r, p ; s}\left(T^{\langle s\rangle} W, f, \frac{a_{n}}{n}\right) .
$$

Proof. We take $g$ as 


$$
\left\|T^{\langle s\rangle} W(f-g)\right\|_{L_{p}(\mathbb{R})}+\delta^{r}\left\|T^{\langle s\rangle} W g^{(r, r, \cdots, r)}\right\|_{L_{p}\left(\mathbb{R}^{s}\right)} \leq C K_{r, p ; s}\left(T^{\langle s\rangle} W ; f, \delta\right),
$$

and for this $g$ we select $P \in \mathcal{P}_{n}\left(\mathbb{R}^{s}\right)$ such that

$$
\|W(g-P)\|_{L^{p}\left(\mathbb{R}^{s}\right)} \leq E_{n, p ; s}(W ; g) .
$$

Then, from Theorem 5.5 we see

$$
\begin{aligned}
E_{n, p ; s}(W, f) & \leq\|W(f-P)\|_{L^{p}\left(\mathbb{R}^{s}\right)} \\
& \leq\|W(f-g)\|_{L^{p}\left(\mathbb{R}^{s}\right)}+\|W(g-P)\|_{L^{p}\left(\mathbb{R}^{s}\right)} \\
& \leq\|W(f-g)\|_{L^{p}\left(\mathbb{R}^{s}\right)}+C E_{n, p ; s}(W, g) \\
& \leq\left\|T^{\langle s\rangle} W(f-g)\right\|_{L^{p}\left(\mathbb{R}^{s}\right)}+C\left(\frac{a_{n}}{n}\right)^{r}\left\|T^{\langle s\rangle} W g^{(r, r, \cdots, r)}\right\|_{L^{p}\left(\mathbb{R}^{s}\right)} \\
& \leq C K_{r, p}\left(T^{\langle s\rangle} W, f, \frac{a_{n}}{n}\right) .
\end{aligned}
$$

Corollary 5.8. Let $1 \leq p \leq \infty$, and let $w \in \mathcal{F}\left(C^{2}+\right)$ be a Freud-type weight. If $\|W f\|_{L_{p}\left(\mathbb{R}^{s}\right)}<\infty$, then we have

$$
E_{n, p ; s}(W, f) \leq C K_{r, p ; s}\left(W, f, \frac{a_{n}}{n}\right) .
$$

Let $0<p \leq \infty$. Damelin [11] gives a K-functional as follows:

$$
\bar{K}_{r, p}\left(f, w, t^{r}\right):=\inf _{P \in \mathcal{P}_{n}}\left\{\|w(f-P)\|_{L^{p}(\mathbb{R})}+t^{r}\left\|P^{(r)} \Phi_{t}^{r} w\right\|_{L^{p}(\mathbb{R})}\right\},
$$

where $t>0$ and $r \geq 1$ are chosen in advance and

$$
n=n(t):=\inf \left\{k ; \frac{a_{k}}{k} \leq t\right\} .
$$

We recall the $r$-th order of the modulus of smoothness $\omega_{r, p}(w ; f ; t)$, which is defined as follows (cf. [6] and [11]). Let $r$ be a positive integer, and let $0<p \leq \infty$. We set

$$
\Delta_{h}^{r}(f, x):=\sum_{i=0}^{r}\left(\begin{array}{l}
r \\
i
\end{array}\right)(-1)^{i} f\left(x+\frac{r h}{2}-i h\right), x \in \mathbb{R} .
$$

For the Freud-type weight,

$$
\bar{\omega}_{r, p}(f, w, t):=\left(\frac{1}{t} \int_{0}^{t}\left\|w \Delta_{h}^{r}(f, x)\right\|_{L^{p}(|x| \leq \sigma(2 t))} \mathrm{d} h\right)^{1 / p}+\inf _{P \in \mathcal{P}_{r-1}}\|(f-P) w\|_{L^{p}(|x| \leq \sigma(4 t))} .
$$

For the Erdös-type weight,

$$
\bar{\omega}_{r, p}(f, w, t):=\left(\frac{1}{t} \int_{0}^{t}\left\|w \Delta_{h \Phi_{t}(x)}^{r}(f, x)\right\|_{L^{p}(|x| \leqslant \sigma(2 t))} \mathrm{d} h\right)^{1 / p}+\inf _{P \in \mathcal{P}_{r-1}}\|(f-P) w\|_{\left.L^{p}(|x| \leq \sigma(t))\right)},
$$

where 


$$
\Phi_{t}(x):=\sqrt{\left|1-\frac{|x|}{\sigma(t)}\right|}+\frac{1}{\sqrt{T(\sigma(t))}} .
$$

We remark that if $T(x)$ is bounded then we see $\Phi_{t}(x) \sim 1$. So, we may consider for only the Erdös-type weight. Then the following proposition holds.

Proposition 5.9 ([11], Theorem 1.2, 1.3). Let $0<p \leq \infty, r \geq 1$, and let $w \in \mathcal{E}_{1}$ (contains $\mathcal{F}\left(C^{2}+\right)$ ). Let $f: \mathbb{R} \rightarrow \mathbb{R}$ for which $w f \in L^{p}(\mathbb{R})$ (for $p=\infty$, we require $f$ to be continuous, and $f w$ to vanish at $\pm \infty)$. Then we have

$$
E_{n, p}(w, f) \leq C_{1} \bar{\omega}_{r, p}\left(f, w, C_{2} \frac{a_{n}}{n}\right) \sim \bar{K}_{r, p}\left(f, w, t^{r}\right) .
$$

On $\mathbb{R}^{s}$ we define

$$
\begin{aligned}
& \Omega_{r, p}(f, W, t):=\max _{i=1, \cdots, s}\left\|W_{i} \bar{\omega}_{r, p}\left(f_{\hat{X}_{i}}, w_{i}, t\right)\right\|_{L^{p}\left(\mathbb{R}^{s-1}\right)}, \\
& \mathcal{K}_{r, p}\left(f, W, t^{r}\right):=\max _{i=1, \cdots, s}\left\|W_{i} \bar{K}_{r, p}\left(f_{\hat{X}_{i}}, w_{i}, t^{r}\right)\right\|_{L^{p}\left(\mathbb{R}^{s-1}\right)} .
\end{aligned}
$$

We see $\Omega_{r, p}(f, W, t) \sim \mathcal{K}_{r, p}\left(f, W, t^{r}\right)$. Then we have the following:

Theorem 5.10. We suppose

$w_{i}=\exp \left(-Q_{i}\right) \in \mathcal{F}_{\lambda}\left(C^{3}+\right)(0<\lambda<3 / 2), i=1,2, \cdots, s$, and let

$$
T_{i}\left(a_{n}\right) \leq c\left(\frac{n}{a_{n}}\right)^{2 / 3}, i=1,2, \cdots, s .
$$

Then

$$
E_{n, p ; s}(W, f) \leq C \Omega_{r, p}\left(f, T^{\langle s\rangle} W, t\right) \sim \mathcal{K}_{r, p}\left(f, T^{\langle s\rangle} W, t^{r}\right) .
$$

Proof. Using

$f-v_{n}^{[s]}=\left(f-v_{n}^{[1]}(f)\right)+\left(v_{n}^{[1]}(f)-v_{n}^{[2]}(f)\right)+\cdots+\left(v_{n}^{[s-1]}(f)-v_{n}^{[s]}(f)\right), \quad$ we get from Proposition 3.3 (2) and (3.2),

$$
\begin{aligned}
& E_{n, p ; s}(W, f) \leq\left\|W\left(f-v_{n}^{[s]}(f)\right)\right\|_{L^{p}\left(\mathbb{R}^{s}\right)} \\
& \leq\left\|W\left(f-v_{n}^{[1]}(f)\right)\right\|_{L^{p}\left(\mathbb{R}^{s}\right)}+\sum_{j=2}^{s}\left\|W\left(v_{n}^{[j-1]}(f)-v_{n}^{[j]}(f)\right)\right\|_{L^{p}\left(\mathbb{R}^{s}\right)} \\
& \leq C\left[\left\|W\left(f-v_{n, 1}(f)\right)\right\|_{L^{p}\left(\mathbb{R}^{s}\right)}+\sum_{j=2}^{s}\left\|W v_{n}^{[j-1]}\left(f-v_{n, j}(f)\right)\right\|_{\left.L^{p}\left(\mathbb{R}^{s}\right)\right]}\right] \\
& \leq C\left[\left\|W\left(f-v_{n, 1}(f)\right)\right\|_{L^{p}\left(\mathbb{R}^{s}\right)}+\sum_{j=2}^{s}\left\|W\left(\prod_{k=1}^{j-1} T_{k}^{1 / 4}\right)\left(f-v_{n, j}(f)\right)\right\|_{\left.L^{p}\left(\mathbb{R}^{s}\right)\right]}\right] \\
& \leq C \sum_{j=1}^{s}\left\|W_{j}\left(\prod_{k=1}^{j-1} T_{k}^{1 / 4}\right) E_{n, p}\left(T_{j}^{1 / 4} w_{j}, f_{\hat{X}_{i}}\right)\right\|_{L^{p}\left(\mathbb{R}_{j}^{s-1}\right)} \leq C \sum_{j=1}^{s}\left\|W_{j}\left(\prod_{1 \leq k \leq s, k \neq j} T_{k}^{1 / 4}\right) \bar{\omega}_{r, p}\left(f_{\hat{X}_{j}}, T_{j}^{1 / 4} w_{j}, \frac{a_{n}}{n}\right)\right\|_{L^{p}\left(\mathbb{R}_{j}^{s-1}\right)} \\
& \sim \sum_{j=1}^{s}\left\|W_{j}\left(\prod_{1 \leq k \leq s, k \neq j} T_{k}^{1 / 4}\right) \bar{K}_{r, p}\left(f_{\hat{X}_{j}}, T_{J}^{1 / j} w_{j},\left(\frac{a_{n}}{n}\right)^{r}\right)\right\|_{L^{p}\left(\mathbb{R}_{j}^{s-1}\right)} \\
& \leq C \Omega_{r, p}\left(f, T^{\langle s\rangle} W, t\right) \sim \mathcal{K}_{r, p}\left(f, T^{\langle s\rangle} W, t^{r}\right) .
\end{aligned}
$$




\section{Approximation for Functions with Bounded Variations}

We define the modulus of continuity. For the Freud-type weight $W$ (all of weights $w_{i}$ are Freud-type), we define

$$
\begin{aligned}
\omega_{p, j}^{*}\left(f_{\hat{X}_{j}}, w_{j}, t\right):= & \sup _{0<h \leq t}\left\|w_{j}(x)\left(\Delta_{h} f_{\hat{X}_{j}}(x)\right)\right\|_{L^{p}\left(|x| \leq \sigma_{j}(2 t)\right)} \\
& +\left\|\left(f_{\hat{X}_{j}}(x)-f_{\hat{X}_{j}}(0)\right) w_{j}(x)\right\|_{L^{p}\left(|x| \leq \sigma_{j}(4 t)\right)} .
\end{aligned}
$$

If $W$ is Erdös-type (some weights $w_{i}$ are Erdös-type), then we define

$$
\begin{aligned}
\omega_{p, j}^{*}\left(f_{\hat{X}_{j}}, w_{j}, t\right):= & \sup _{0<h \leq t}\left\|w_{j}(x)\left(\Delta_{h \Phi_{t, j}(x)} f_{\hat{X}_{j}}(x)\right)\right\|_{L^{p}\left(|x| \leq \sigma_{j}(2 t)\right)} \\
& +\left\|\left(f_{\hat{X}_{j}}(x)-f_{\hat{X}_{j}}(0)\right) w_{j}(x)\right\|_{L^{p}\left(|x| \leq \sigma_{j}(4 t)\right)} .
\end{aligned}
$$

It is sufficient to consider only the modulus for the Erdös-type.

Assumption 6.1. Let $w_{i} \in \mathcal{F}\left(C^{2}+\right)(i=1,2, \cdots, s)$, and let $a_{n}^{(i)} \sim a_{n}, i=1,2, \cdots, s$. Suppose that $f$ is continuous on $\mathbb{R}^{s}$, and $f_{\hat{x}_{i}}(x)$ has a bounded variation on any compact interval in $\mathbb{R}^{s}$ with

$$
\int_{\mathbb{R}_{i}^{s-1}} W_{i} \int_{\mathbb{R}} w_{i}(x)\left|\mathrm{d} f_{\hat{x}_{i}}(x)\right| \mathrm{d} x_{1} \cdots \mathrm{d}_{x_{i-1}} \mathrm{~d} x_{i+1} \cdots \mathrm{d} x_{s}<\infty, \quad i=1,2, \cdots, s,
$$

and if $p=\infty$, then we further suppose

$$
\left|W(X) f_{\hat{X}_{i}}\left(x_{i}\right)\right| \rightarrow 0 \text {, as }\left|x_{i}\right| \rightarrow \infty, i=1,2, \cdots, s .
$$

In (6.7) and (6.8) below, we put $t=a_{n} / n$, where $a_{n} \sim a_{n}^{(i)}, i=1,2, \cdots, s$. Especially, in (6.8) we set

$$
\left\|W_{i} \omega_{\infty, i}^{*}\left(f_{\hat{X}_{i}}, w_{i} ; \frac{a_{n}^{(i)}}{n}\right)\right\|_{L^{\infty}\left(\mathbb{R}_{i}^{s-1}\right)}=o_{n}(1) .
$$

Theorem 6.2. We suppose $w_{j}=\exp \left(-Q_{j}\right) \in \mathcal{F}\left(C^{2}+\right), j=1,2, \cdots, s$, and let (3.5) hold. Let $n \geq 1$. Then we have

$$
\left\|\frac{W\left(f-v_{n}^{[s]}(f)\right)}{\prod_{k=1}^{s} T_{k}^{1 / 4}}\right\|_{L^{1}\left(\mathbb{R}^{s}\right)} \leq \sum_{j=1}^{s} C_{j}\left\|W_{j} \omega_{p, j}^{*}\left(f_{\hat{X}_{j}}, w_{j} ; c_{j} \frac{a_{n}^{(j)}}{n}\right)\right\|_{L^{p}\left(\mathbb{R}_{j}^{s-1}\right)} .
$$

Now Assumption 6.1 holds. Then we have

$$
\sum_{j=1}^{s} C_{j}\left\|W_{j} \omega_{p, j}^{*}\left(f_{\hat{X}_{j}}, w_{j} ; C_{j} \frac{a_{n}^{(j)}}{n}\right)\right\|_{L^{p}\left(\mathbb{R}_{j}^{s-1}\right)} \leq \operatorname{Co}_{n}(1)^{1-1 / p}\left(\frac{a_{n}}{n}\right)^{\frac{1}{p}} .
$$

Proof of (6.4). By Proposition 3.3 (1) and Proposition 3.4, we get

$$
\left\|\frac{w_{1}(x)\left(f_{\hat{X}_{1}}-v_{n, 1}\left(f_{\hat{X}_{1}}\right)\right)}{T_{1}^{1 / 4}(x)}\right\|_{L^{p}(\mathbb{R})} \leq C E_{1, n}\left(f_{\hat{X}_{1}}, w_{1}\right) \leq C_{1} \omega_{p, 1}^{*}\left(f_{\hat{X}_{1}}, w_{1} ; c_{1} \frac{a_{n}^{(1)}}{n}\right),
$$


where the constant $C_{1}$ and $c_{1}$ is independent of $\hat{X}_{1}$. Similarly, for $j=1,2, \cdots, s$,

$$
\left\|\frac{w_{j}(x)\left(f_{\hat{X}_{j}}-v_{n, j}\left(f_{\hat{X}_{j}}\right)\right)}{T_{j}^{1 / 4}(x)}\right\|_{L^{p}(\mathbb{R})} \leq C_{j} \omega_{p, j}^{*}\left(f_{\hat{x}_{j}}, w_{j} ; c_{j} \frac{a_{n}^{(j)}}{n}\right) .
$$

Using $f-v_{n}^{[s]}=\left(f-v_{n}^{[1]}(f)\right)+\left(v_{n}^{[1]}(f)-v_{n}^{[2]}(f)\right)+\cdots+\left(v_{n}^{[s-1]}(f)-v_{n}^{[s]}(f)\right)$, we get from Lemma 3.7 (1) and (6.6),

$$
\begin{aligned}
& \left\|\frac{W\left(f-v_{n}^{[s]}(f)\right)}{T^{\langle s\rangle}}\right\|_{L^{p}\left(\mathbb{R}^{s}\right)} \\
& \leq\left\|\frac{W\left(f-v_{n}^{[1]}(f)\right)}{T^{\langle 1\rangle}}\right\|_{L_{p}\left(\mathbb{R}^{s}\right)}+\sum_{j=2}^{s}\left\|\frac{W\left(v_{n}^{[j-1]}(f)-v_{n}^{[j]}(f)\right)}{T^{\langle j\rangle}}\right\|_{L^{p}\left(\mathbb{R}^{s}\right)} \\
& \leq C\left[\left\|\frac{W\left(f-v_{n, 1}(f)\right)}{T_{1}^{1 / 4}}\right\|_{L^{p}\left(\mathbb{R}^{s}\right)}+\sum_{j=2}^{s}\left\|\frac{W v_{n}^{[j-1]}\left(\left\{f-v_{n, j}(f)\right\}\right) / T_{j}^{1 / 4}}{T^{(j-1\rangle}}\right\|_{L^{p}\left(\mathbb{R}^{s}\right)}\right] \\
& \leq C\left[\left\|\frac{W\left(f-v_{n, 1}(f)\right)}{T_{1}^{1 / 4}}\right\|_{L^{p}\left(\mathbb{R}^{s}\right)}+\sum_{j=2}^{s}\left\|\frac{W\left(f-v_{n, j}(f)\right)}{T_{j}^{1 / 4}}\right\|_{\left.L^{p}\left(\mathbb{R}^{s}\right)\right]}\right] \\
& \leq C_{1}\left\|W_{1} \omega_{p, 1}^{*}\left(f_{\hat{X}_{1}}, w_{1} ; c_{1} \frac{a_{n}^{(1)}}{n}\right)\right\|_{L^{p}\left(\mathbb{R}_{1}^{s-1}\right)}+\sum_{j=2}^{s} C_{j}\left\|W_{j} \omega_{p, j}^{*}\left(f_{\hat{X}_{j}}, w_{j} ; C_{j} \frac{a_{n}^{(j)}}{n}\right)\right\|_{L^{p}\left(\mathbb{R}_{j}^{s-1}\right)} \\
& \leq \sum_{j=1}^{s} C_{j}\left\|\left(\prod_{1 \leq i \leq s, i \neq j} w_{i}\right) \omega_{p, j}^{*}\left(f_{\hat{X}_{j}}, w_{j} ; c_{j} \frac{a_{n}^{(j)}}{n}\right)\right\|_{L^{p}\left(\mathbb{R}_{j}^{s-1}\right)} \cdot
\end{aligned}
$$

Hence, we have (6.4). \#

To prove Theorem 6.2 (6.5) we need the following two theorems.

Theorem 6.3 (cf. [12], Proposition 3.2). Let $W=\prod_{i=1}^{s} w_{i}, w_{i} \in \mathcal{F}\left(C^{2}+\right)$, $i=1,2, \cdots, s$. Let $f$ hold Assumption 6.1, especially (6.1) and (6.2) hold. Then there exists a constant $C>0$ such that for every $t>0$ and $i=1,2, \cdots, s$,

$$
\begin{aligned}
& \left\|W_{i} \omega_{1, i}^{*}\left(f_{\hat{X}_{i}}, w_{i} ; t\right)\right\|_{L^{1}\left(\mathbb{R}_{i}^{s-1}\right)} \\
& \leq C t \int_{\mathbb{R}_{i}^{s-1}} W_{i} \int_{\mathbb{R}} w_{i}(x)\left|\mathrm{d} f_{\hat{X}_{i}}(x)\right| \mathrm{d} x_{1} \cdots \mathrm{d} x_{i-1} \mathrm{~d} x_{i+1} \cdots \mathrm{d} x_{s}
\end{aligned}
$$

and

$$
\lim _{t \rightarrow 0}\left\|W_{i} \omega_{\infty, i}^{*}\left(f_{\hat{X}_{i}}, w_{i} ; t\right)\right\|_{L^{\infty}\left(\mathbb{R}_{i}^{s-1}\right)}=0 .
$$

Proof. Let $g_{\hat{X}_{i}}(x):=f_{\hat{X}_{i}}(x)-f_{\hat{X}_{i}}(0)$. For $t>0$, we write $\sigma_{i}(t)=a_{u}^{(i)}$, and let $0<h \leq t$ and $\left|x=x_{i}\right| \leq \sigma_{i}(2 t)<a_{u}^{(i)}$. Since $\Phi_{t}(x) \leq 2$ for $|x| \leq \sigma_{i}(2 t)$, if we take $t$ small enough, then 


$$
\begin{aligned}
& \int_{|x| \leq \sigma_{i}(2 t)}\left|\left\{g_{\hat{X}_{i}}\left(x+\frac{h}{2} \Phi_{t}(x)\right)-g_{\hat{X}_{i}}\left(x-\frac{h}{2} \Phi_{t}(x)\right)\right\} w(x)\right| \mathrm{d} x \\
& \leq \int_{|x| \leq \sigma_{i}(2 t)} \int_{x-\frac{h}{2} \Phi_{t}(x)}^{x+\frac{h}{2} \Phi_{t}(x)} w_{i}(y)\left|\mathrm{d} g_{\hat{X}_{i}}(y)\right| \mathrm{d} x \\
& \leq \int_{\mathbb{R}} \int_{x-h}^{x+h} w_{i}(y)\left|\mathrm{d} g_{\hat{X}_{i}}(y)\right| \mathrm{d} x \\
& \leq \int_{\mathbb{R}} w_{i}(y) \int_{y-h}^{y+h} \mathrm{~d} x\left|\mathrm{~d} g_{\hat{X}_{i}}(y)\right| \leq h \int_{\mathbb{R}} w_{i}(y)\left|\mathrm{d} f_{\hat{X}_{i}}(y)\right| .
\end{aligned}
$$

On the other hand, by Proposition 4.2 with $p=1$,

$$
\begin{aligned}
& \int_{\sigma_{i}(4 t)}^{\infty}\left|\left\{f_{\hat{X}_{i}}(x)-f_{\hat{X}_{i}}(0)\right\} w_{i}(x)\right| \mathrm{d} x \\
& \leq \frac{1}{Q_{i}^{\prime}\left(\sigma_{i}(4 t)\right)} \int_{\sigma_{i}(4 t)}^{\infty} Q_{i}^{\prime}(x)\left|g_{\hat{X}_{i}}(x)\right| w_{i}(x) \mathrm{d} x \\
& \leq C t \int_{0}^{\infty} Q_{i}^{\prime}\left(x_{i}\right) w_{i}\left(x_{i}\right) \int_{0}^{x}\left|\mathrm{~d} f_{\hat{X}_{i}}(y)\right| \mathrm{d} x_{i} \\
& \leq C t \int_{0}^{\infty}\left(\int_{y}^{\infty} Q_{i}^{\prime}\left(x_{i}\right) w_{i}\left(x_{i}\right) \mathrm{d} x\right)\left|\mathrm{d} f_{\hat{X}_{i}}(y)\right| \\
& =C t \int_{0}^{\infty} w_{i}(y)\left|\mathrm{d} f_{\hat{X}_{i}}(y)\right| .
\end{aligned}
$$

Similarly,

$$
\int_{-\infty}^{-\sigma_{i}(4 t)}\left|\left\{f_{\hat{X}_{i}}(x)-f_{\hat{X}_{i}}(0)\right\} w_{i}(x)\right| \mathrm{d} x \leq C t \int_{-\infty}^{0} w_{i}(y)\left|\mathrm{d} f_{\hat{X}_{i}}(y)\right| .
$$

Therefore, we see

$$
\int_{-\infty}^{\infty}\left|\left\{f_{\hat{X}_{i}}(x)-f_{\hat{X}_{i}}(0)\right\} w_{i}(x)\right| \mathrm{d} x \leq C t \int_{-\infty}^{\infty} w_{i}(y)\left|\mathrm{d} f_{\hat{X}_{i}}(y)\right| .
$$

Consequently, from (6.9) and (6.10) we have

$$
\begin{aligned}
& \left\|W_{i} \omega_{1, i}^{*}\left(f_{\hat{X}_{i}}, w_{i} ; t\right)\right\|_{L^{1}\left(\mathbb{R}_{i}^{s-1}\right)} \\
& \leq C t \int_{\mathbb{R}_{i}^{s-1}} W_{i} \int_{\mathbb{R}} W_{i}(x)\left|\mathrm{d} f_{\hat{X}_{i}}(x)\right| \mathrm{d} x_{1} \cdots \mathrm{d} x_{i-1} \mathrm{~d} x_{i+1} \cdots \mathrm{d} x_{s},
\end{aligned}
$$

that is, we have (6.7). We show (6.8). Let $\varepsilon>0,|x| \geq L$ for large $L>0$. Then

$$
\begin{aligned}
& \left|W_{i} w_{i}(x)\left(f_{\hat{X}_{i}}\left(x+\frac{h}{2} \Phi_{t}(x)\right)-f_{\hat{X}_{i}}\left(x-\frac{h}{2} \Phi_{t}(x)\right)\right)\right| \\
& \leq 2 C\left|W_{i} w_{i}(x) f_{\hat{X}_{i}}(x)\right| \leq 2 C \varepsilon .
\end{aligned}
$$

Next, for $|x| \leq L$ we see

$$
\left|W_{i} w_{i}(x)\left(f_{\hat{X}_{i}}\left(x+\frac{h}{2} \Phi_{t}(x)\right)-f_{\hat{X}_{i}}\left(x-\frac{h}{2} \Phi_{t}(x)\right)\right)\right| \rightarrow 0 \text { as } t \rightarrow 0,
$$

hence for $0<t \leq t_{0}$ small enough,

$$
\left|W_{i} w_{i}(x)\left(f_{\hat{X}_{i}}\left(x+\frac{h}{2} \Phi_{t}(x)\right)-f_{\hat{X}_{i}}\left(x-\frac{h}{2} \Phi_{t}(x)\right)\right)\right| \leq \varepsilon .
$$

On the other hand, 


$$
\begin{aligned}
& \left\|W_{i} w_{i}(x)\left(f_{\hat{X}_{i}}(x)-f_{\hat{X}_{i}}(0)\right)\right\|_{L^{\infty}\left(|x| \geq \sigma_{i}(4 t)\right)} \\
& \leq\left\|W_{i} w_{i}(x) f_{\hat{X}_{i}}(x)\right\|_{L^{\infty}\left(|x| \geq \sigma_{i}(4 t)\right)}+\left|f_{\hat{X}_{i}}(0)\right|\left\|W_{i} w_{i}(x)\right\|_{L^{\infty}\left(|x| \geq \sigma_{i}(4 t)\right)} \rightarrow 0 \text { as } t \rightarrow 0 .
\end{aligned}
$$

Hence for $0<t \leq t_{0}$ small enough,

$$
\left\|W(X)\left(f_{\hat{X}_{i}}(x)-f_{\hat{X}_{i}}(0)\right)\right\|_{L^{\infty}\left(|x| \geq \sigma_{i}(4 t)\right)} \leq \varepsilon .
$$

Consequently, we have

$$
\lim _{t \rightarrow 0}\left\|W_{i} \omega_{\infty, i}^{*}\left(f_{\hat{X}_{i}}, w_{i} ; t\right)\right\|_{L^{\infty}\left(\mathbb{R}_{i}^{s-1}\right)}=0,
$$

that is, (6.8). \#

Theorem 6.4. Under Assumption 6.1, we have

$$
\int_{\mathbb{R}_{i}^{s-1}}\left|W_{i} \omega_{p, i}^{*}\left(f_{\hat{X}_{i}}, w_{i}: t\right)\right|^{p} D X_{i} \leq C o_{t}(1)^{p-1} t,
$$

where

$$
D X_{i}:=\mathrm{d} x_{1} \cdots \mathrm{d} x_{i-1} \mathrm{~d} x_{i+1} \cdots \mathrm{d} x_{s}, i=1,2, \cdots, s
$$

and

$$
o_{t}(1):=\left\|W_{i} \omega_{\infty, i}^{*}\left(f_{\hat{X}_{i}}, w_{i} ; t\right)\right\|_{L^{\infty}\left(\mathbb{R}_{i}^{s-1}\right)} .
$$

Proof. Let $g_{\hat{X}_{i}}(x):=f_{\hat{X}_{i}}(x)-f_{\hat{X}_{i}}(0)$.

$$
\begin{aligned}
& \int_{\left|x_{i}\right| \leq \sigma_{i}(2 t)}\left|\left\{g_{\hat{X}_{i}}\left(x_{i}+\frac{h}{2} \Phi_{t}\left(x_{i}\right)\right)-g_{\hat{X}_{i}}\left(x_{i}-\frac{h}{2} \Phi_{t}\left(x_{i}\right)\right)\right\} w_{i}\left(x_{i}\right)\right|^{p} \mathrm{~d} x_{i} \\
& \leq\left[\sup _{\left|x_{i}\right| \leq \sigma_{i}(2 t)}\left|\left\{g_{\hat{X}_{i}}\left(x_{i}+\frac{h}{2} \Phi_{t}\left(x_{i}\right)\right)-g_{\hat{X}_{i}}\left(x_{i}-\frac{h}{2} \Phi_{t}\left(x_{i}\right)\right)\right\} w_{i}\left(x_{i}\right)\right|^{p-1}\right. \\
& +\sup _{\left|x_{i}\right| \geq \sigma_{i}(4 t)}\left\{\left.\left\{f_{\hat{X}_{i}}\left(x_{i}\right)-f_{\hat{X}_{i}}(0)\right\} w_{i}\left(x_{i}\right)\right|^{p-1}\right] \\
& \times\left[\int_{\left|x_{i}\right| \leq \sigma_{i}(2 t)}\left|\left\{g_{\hat{X}_{i}}\left(x_{i}+\frac{h}{2} \Phi_{t}\left(x_{i}\right)\right)-g_{\hat{X}_{i}}\left(x_{i}-\frac{h}{2} \Phi_{t}\left(x_{i}\right)\right)\right\} w_{i}\left(x_{i}\right)\right| \mathrm{d} x_{i}\right. \\
& \left.+\int_{\left|x_{i}\right| \geq \sigma_{i}(4 t)}\left|\left\{f_{\hat{X}_{i}}\left(x_{i}\right)-f_{\hat{X}_{i}}(0)\right\} w_{i}\left(x_{i}\right)\right| \mathrm{d} x_{i}\right] \\
& \leq\left[\sup _{\left|x_{i}\right| \leq \sigma_{i}(2 t)}\left|\left\{g_{\hat{X}_{i}}\left(x_{i}+\frac{h}{2} \Phi_{t}\left(x_{i}\right)\right)-g_{\hat{X}_{i}}\left(x_{i}-\frac{h}{2} \Phi_{t}\left(x_{i}\right)\right)\right\} w_{i}\left(x_{i}\right)\right|\right. \\
& +\sup _{\left|x_{i}\right| \geq \sigma_{i}(4 t)}\left\{\left\{f_{\hat{X}_{i}}\left(x_{i}\right)-f_{\hat{X}_{i}}(0)\right\} w_{i}\left(x_{i}\right) \mid\right]^{p-1} \\
& \times\left[\int_{\left|x_{i}\right| \leq \sigma_{i}(2 t)}\left|\left\{g_{\hat{X}_{i}}\left(x_{i}+\frac{h}{2} \Phi_{t}\left(x_{i}\right)\right)-g_{\hat{X}_{i}}\left(x_{i}-\frac{h}{2} \Phi_{t}\left(x_{i}\right)\right)\right\} w_{i}\left(x_{i}\right)\right| \mathrm{d} x_{i}\right. \\
& \left.+\int_{\left|x_{i}\right| \geq \sigma_{i}(4 t)}\left|\left\{f_{\hat{X}_{i}}\left(x_{i}\right)-f_{\hat{X}_{i}}(0)\right\} w_{i}\left(x_{i}\right)\right| \mathrm{d} x_{i}\right] \\
& =\omega_{\infty, i}^{*}\left(f_{\hat{X}_{i}}, w_{i} ; t\right)^{p-1} \times \omega_{1, i}^{*}\left(f_{\hat{X}_{i}}, w_{i} ; t\right) \text {. }
\end{aligned}
$$


Similarly,

$$
\begin{aligned}
& \int_{\left|x_{i}\right| \geq \sigma_{i}(4 t)}\left|\left\{f_{\hat{X}_{i}}\left(x_{i}\right)-f_{\hat{X}_{i}}(0)\right\} w_{i}\left(x_{i}\right)\right|^{p} \mathrm{~d} x_{i} \\
& \leq\left[\sup _{\left|x_{i}\right| \geq \sigma_{i}(4 t)}\left|\left\{f_{\hat{X}_{i}}\left(x_{i}\right)-f_{\hat{X}_{i}}(0)\right\} w_{i}\left(x_{i}\right)\right|^{p-1}\right. \\
&\left.+\sup _{\left|x_{i}\right| \leq \sigma_{i}(2 t)}\left|\left\{g_{\hat{X}_{i}}\left(x_{i}+\frac{h}{2} \Phi_{t}\left(x_{i}\right)\right)-g_{\hat{X}_{i}}\left(x_{i}-\frac{h}{2} \Phi_{t}\left(x_{i}\right)\right)\right\} w_{i}\left(x_{i}\right)\right|^{p-1}\right] \\
& \quad \times\left[\sup _{\left|x_{i}\right| \geq \sigma_{i}(4 t)}\left|\left\{f_{\hat{X}_{i}}\left(x_{i}\right)-f_{\hat{X}_{i}}(0)\right\} w_{i}\left(x_{i}\right)\right|\right. \\
&\left.+\int_{\left|x_{i}\right| \leq \sigma_{i}(2 t)}\left|\left\{g_{\hat{X}_{i}}\left(x_{i}+\frac{h}{2} \Phi_{t}\left(x_{i}\right)\right)-g_{\hat{X}_{i}}\left(x_{i}-\frac{h}{2} \Phi_{t}\left(x_{i}\right)\right)\right\} w_{i}\left(x_{i}\right)\right| \mathrm{d} x_{i}\right] \\
& \leq\left[\sup _{\left|x_{i}\right| \geq \sigma_{i}(4 t)}\left|\left\{f_{\hat{X}_{i}}\left(x_{i}\right)-f_{\hat{X}_{i}}(0)\right\} w_{i}\left(x_{i}\right)\right|\right. \\
&\left.\left.+\sup _{\left|x_{i}\right| \leq \sigma_{i}(2 t)}\left|\left\{g_{\hat{X}_{i}}\left(x_{i}+\frac{h}{2} \Phi_{t}\left(x_{i}\right)\right)-g_{\hat{X}_{i}}\left(x_{i}-\frac{h}{2} \Phi_{t}\left(x_{i}\right)\right)\right\} w_{i}\left(x_{i}\right)\right|\right]\right]^{p-1} \\
& \times\left[\int_{\left|x_{i}\right| \geq \sigma_{i}(4 t)}\left|\left\{f_{\hat{X}_{i}}\left(x_{i}\right)-f_{\hat{X}_{i}}(0)\right\} w_{i}\left(x_{i}\right)\right| \mathrm{d} x_{i}\right. \\
&\left.+\int_{\left|x_{i}\right| \leq \sigma_{i}(2 t)}\left|\left\{g_{\hat{X}_{i}}\left(x_{i}+\frac{h}{2} \Phi_{t}\left(x_{i}\right)\right)-g_{\hat{X}_{i}}\left(x_{i}-\frac{h}{2} \Phi_{t}\left(x_{i}\right)\right)\right\} w_{i}\left(x_{i}\right)\right| \mathrm{d} x_{i}\right] \\
&=\left.\omega_{\infty \infty, i}^{*}\left(f_{\hat{X}_{i}}, w_{i} ; t\right)\right|^{p-1} \times \omega_{1, i}^{*}\left(f_{\hat{X}_{i}}, w_{i} ; t\right) .
\end{aligned}
$$

Hence,

$$
\omega_{p, i}^{*}\left(f_{\hat{X}_{i}}, w_{i}, t\right) \leq C\left(\omega_{\infty, i}^{*}\left(f_{\hat{X}_{i}}, w_{i}, t\right)^{p-1} \times \omega_{1, i}^{*}\left(f_{\hat{X}_{i}}, w_{i}, t\right)\right),
$$

Consequently, we have

$$
\begin{aligned}
& \int_{\mathbb{R}_{i}^{s-1}}\left|W_{i} \omega_{p, i}^{*}\left(f_{\hat{X}_{i}}, w_{i} ; t\right)\right|^{p} D X_{i} \\
& \leq C \int_{\mathbb{R}_{i}^{s-1}}\left\{W_{i} \omega_{\infty, i}^{*}\left(f_{\hat{X}_{i}}, w_{i} ; t\right)\right\}^{p-1}\left\{W_{i} \omega_{1, i}^{*}\left(f_{\hat{X}_{i}}, w_{i} ; t\right)\right\} D X_{i} \\
& \leq C\left\|W_{i} \omega_{\infty, i}^{*}\left(f_{\hat{X}_{i}}, w_{i} ; t\right)\right\|_{L^{\infty}\left(\mathbb{R}_{i}^{s-1}\right)}^{p-1} \int_{\mathbb{R}_{i}^{s-1}} W_{i} \omega_{1, i}^{*}\left(f_{\hat{X}_{i}}, w_{i} ; t\right) D X_{i} \\
& \leq C o_{t}(1)^{p-1} t,
\end{aligned}
$$

where

$$
o_{t}(1):=\left\|W_{i} \omega_{\infty, i}^{*}\left(f_{\hat{X}_{i}}, w_{i} ; t\right)\right\|_{L^{\infty}\left(\mathbb{R}_{i}^{s-1}\right)} \cdot \quad \#
$$

Proof of Theorem 6.2 (6.5). Using Theorems 6.3 and 6.4 with $t=a_{n} / n$, we easily obtain (6.5).

We will give an analogy of Theorem 6.2. To do so we use the following weights . They are guaranteed by Proposition 2.3. 


$$
\begin{aligned}
& w_{i}\left(x_{i}\right) T_{i}^{1 / 4}\left(x_{i}\right) \sim w_{i}^{\$} \in \mathcal{F}\left(C^{2}+\right), i=1,2, \cdots, s, \\
& W^{\S}:=\prod_{i=1}^{s} w_{i}^{\$}, W_{i}^{\$}:=\prod_{1 \leq j \leq s, j \neq i} w_{j}^{\$} .
\end{aligned}
$$

Assumption 6.5. Let $w_{i} \in \mathcal{F}\left(C^{3}+\right), 0<\lambda<3 / 2(i=1, \cdots, s)$. Suppose that $f$ is continuous on $\mathbb{R}^{s}$, and $f_{\hat{x}_{i}}(x)$ has a bounded variation on any compact interval in $\mathbb{R}^{s}$ with

$$
\int_{\mathbb{R}_{i}^{s-1}} W_{i}^{\$} \int_{\mathbb{R}} w_{i}^{\$}(x)\left|\mathrm{d} f_{\hat{X}_{i}}(x)\right| \mathrm{d} x_{1} \cdots \mathrm{d}_{x_{i-1}} \mathrm{~d} x_{i+1} \cdots \mathrm{d} x_{s}<\infty, \quad i=1,2, \cdots, s,
$$

and if $p=\infty$, then we further suppose

$$
\left|W^{\$}(X) f_{\hat{X}_{i}}\left(x_{i}\right)\right| \rightarrow 0 \text {, as }\left|x_{i}\right| \rightarrow \infty, i=1,2, \cdots, s,
$$

where the weights $w_{i}^{\$}, W^{\$}$ and $W_{i}^{\$}$ are defined by (6.11).

Theorem 6.6. We suppose

$$
\begin{gathered}
w_{j}=\exp \left(-Q_{j}\right) \in \mathcal{F}_{\lambda}\left(C^{3}+\right)(0<\lambda<3 / 2), j=1,2, \cdots, s, \text { and let } \\
T_{j}\left(a_{n}^{(j)}\right) \leq c\left(\frac{n}{a_{n}^{(j)}}\right)^{\frac{2}{3}}, j=1,2, \cdots, s .
\end{gathered}
$$

Let $n \geq 1,1 \leq p \leq \infty$. Then we have

$$
\begin{aligned}
& \left\|W\left(f-v_{n}^{(s)}(f)\right)\right\|_{L^{p}\left(\mathbb{R}^{s}\right)} \\
& \leq \sum_{j=1}^{s} C_{j}\left\|W_{j}\left(\prod_{k=1}^{j-1} T_{k}^{1 / 4}\right) \omega_{p, j}^{*}\left(f_{\hat{X}_{j}}, T_{j}^{1 / 4} w_{j} ; c_{j} \frac{a_{n}^{(j)}}{n}\right)\right\|_{L^{p}\left(\mathbb{R}_{j}^{s-1}\right)} .
\end{aligned}
$$

Now Assumption 6.5 holds. Then we have

$$
\begin{aligned}
& \sum_{j=1}^{s} C_{j}\left\|W_{j}\left(\prod_{k=1}^{j-1} T_{k}^{1 / 4}\right) \omega_{p, j}^{*}\left(f_{\hat{X}_{j}}, T_{j}^{1 / 4} w_{j} ; c_{j} \frac{a_{n}^{(j)}}{n}\right)\right\|_{L^{p}\left(\mathbb{R}_{j}^{s-1}\right)} \\
& \leq C o_{n}(1)^{1-1 / p}\left(\frac{a_{n}}{n}\right)^{\frac{1}{p}} .
\end{aligned}
$$

Proof of (6.15). By Proposition 3.3 (2) and Proposition 3.4, we get

$$
\begin{aligned}
& \left\|w_{1}(x)\left(f_{\hat{X}_{1}}-v_{n, 1}\left(f_{\hat{X}_{1}}\right)\right)\right\|_{L^{p}(\mathbb{R})} \\
& \leq C E_{p, n}\left(f_{\hat{X}_{1}} ; T_{1}^{1 / 4} w_{1}\right) \leq C_{1} \omega_{p, 1}^{*}\left(f_{\hat{X}_{1}}, T_{1}^{1 / 4} w_{1} ; c_{1} \frac{a_{n}^{(1)}}{n}\right),
\end{aligned}
$$

where the constant $C_{1}$ and $c_{1}$ are independent of $\hat{X}_{1}$. Similarly, for $j=1,2, \cdots, s$,

$$
\left\|w_{j}(x)\left(f_{\hat{X}_{j}}-v_{n, j}\left(f_{\hat{X}_{j}}\right)\right)\right\|_{L^{p}(\mathbb{R})} \leq C_{j} \omega_{p, j}^{*}\left(f_{\hat{X}_{j}}, T_{j}^{1 / 4} w_{j} ; c_{j} \frac{a_{n}^{(j)}}{n}\right) .
$$

Using $\quad f-v_{n}^{[s]}=\left(f-v_{n}^{[1]}(f)\right)+\left(v_{n}^{[1]}(f)-v_{n}^{[2]}(f)\right)+\cdots+\left(v_{n}^{[s-1]}(f)-v_{n}^{[s]}(f)\right)$, 
we get from Lemma 3.7 (2) and (6.17),

$$
\begin{aligned}
& \left\|W\left(f-v_{n}^{[s]}(f)\right)\right\|_{L^{p}\left(\mathbb{R}^{s}\right)} \\
& \leq\left\|W\left(f-v_{n}^{[1]}(f)\right)\right\|_{L^{p}\left(\mathbb{R}^{s}\right)}+\sum_{j=2}^{s}\left\|W\left(v_{n}^{[j-1]}(f)-v_{n}^{[j]}(f)\right)\right\|_{L^{p}\left(\mathbb{R}^{s}\right)} \\
& \leq C\left[\left\|W\left(f-v_{n, 1}(f)\right)\right\|_{L^{p}\left(\mathbb{R}^{s}\right)}+\sum_{j=2}^{s}\left\|W v_{n}^{[j-1]}\left(f-v_{n, j}(f)\right)\right\|_{\left.L^{p}\left(\mathbb{R}^{s}\right)\right]}\right. \\
& \leq C\left[\left\|W\left(f-v_{n, 1}(f)\right)\right\|_{L^{p}\left(\mathbb{R}^{s}\right)}+\sum_{j=2}^{s}\left\|W\left(\prod_{k=1}^{j-1} T_{k}^{1 / 4}\right)\left(f-v_{n, j}(f)\right)\right\|_{\left.L^{p}\left(\mathbb{R}^{s}\right)\right]}\right] \\
& \leq C_{1}\left\|W_{1} \omega_{p, 1}^{*}\left(f_{\hat{X}_{1}}, T_{1}^{1 / 4} W_{1} ; c_{1} \frac{a_{n}^{(1)}}{n}\right)\right\|\left\|_{L^{p}\left(\mathbb{R}_{j}^{s-1}\right)}{ }^{s}\right\| W_{j}\left(\prod_{k=1}^{j-1} T_{k}^{1 / 4}\right) \omega_{p, j}^{*}\left(f_{\hat{X}_{j}}, T_{j}^{1 / 4} w_{j} ; c_{j} \frac{a_{n}^{(j)}}{n}\right) \|_{L^{p}\left(\mathbb{R}_{j}^{s-1}\right)}
\end{aligned}
$$

by (3.11)

$$
\leq \sum_{j=1}^{s} C_{j}\left\|W_{j}\left(\prod_{k=1}^{j-1} T_{k}^{1 / 4}\right) \omega_{p, j}^{*}\left(f_{\hat{X}_{j}}, T_{j}^{1 / 4} w_{j} ; c_{j} \frac{a_{n}^{(j)}}{n}\right)\right\|_{L^{p}\left(\mathbb{R}_{j}^{s-1}\right)},
$$

where $\prod_{k=1}^{j-1} T_{k}^{1 / 4}=1$ for $j=1$, that is, we have (6.15).

(6.16) follows from the following theorems. \#

In Theorems 6.3 and 6.4 we replace $w_{i}, W$ with $w_{i}^{\$}, W^{\$}$ of $(6.11)$, where $w_{i} \in \mathcal{F}_{\lambda}\left(C^{3}+\right), 0<\lambda<3 / 2(i=1,2, \cdots, s)$, then we easily have the following Theorem 6.7.

Theorem 6.7 (cf. [Theorem 6.3 in this paper]). Let $W^{\$}=\prod_{i=1}^{s} w_{i}^{\$}, w_{i}^{\$} \in \mathcal{F}\left(C^{2}+\right), i=1,2, \cdots, s$. Let $f$ hold Assumption 6.5, especially (6.18) and (6.19) hold. Then there exists a constant $C>0$ such that for every $t>0$ and $i=1,2, \cdots, s$,

$$
\begin{aligned}
& \left\|W_{i}^{\$} \omega_{1, i}^{*}\left(f_{\hat{X}_{i}}, w_{i}^{\$} ; t\right)\right\|_{L^{1}\left(\mathbb{R}_{i}^{s-1}\right)} \\
& \leq C t \int_{\mathbb{R}_{i}^{s-1}} W_{i}^{\$} \int_{\mathbb{R}} w_{i}^{\$}(x)\left|\mathrm{d} f_{\hat{X}_{i}}(x)\right| \mathrm{d} x_{1} \cdots \mathrm{d} x_{i-1} \mathrm{~d} x_{i+1} \cdots \mathrm{d} x_{s},
\end{aligned}
$$

and

$$
\lim _{t \rightarrow 0}\left\|W_{i}^{\$} \omega_{\infty, i}^{*}\left(f_{\hat{X}_{i}}, w_{i}^{\$} ; t\right)\right\|_{L^{\infty}\left(\mathbb{R}_{i}^{s-1}\right)}=0 .
$$

Theorem 6.8. Under Assumption 6.5, we have

$$
\int_{\mathbb{R}_{i}^{s-1}}\left|W_{i}^{\$} \omega_{p, i}^{*}\left(f_{\hat{X}_{i}}, w_{i}^{\$} ; t\right)\right|^{p} D X_{i} \leq \operatorname{Co}_{t}(1)^{p-1} t,
$$

where

$$
D X_{i}:=\mathrm{d} x_{1} \cdots \mathrm{d} x_{i-1} \mathrm{~d} x_{i+1} \cdots \mathrm{d} x_{s}, i=1,2, \cdots, s
$$


and

$$
o_{t}(1):=\left\|W_{i}^{\$} \omega_{\infty, i}^{*}\left(f_{\hat{X}_{i}}, w_{i}^{\$} ; t\right)\right\|_{L^{\infty}\left(\mathbb{R}_{i}^{s-1}\right)} .
$$

\section{Approximation for Functions of the Lipschitz Class}

Through this section we consider the weight $w=\exp (-Q) \in \mathcal{F}_{\lambda}\left(C^{3}+\right)$.

Theorem 7.1. (1) We suppose $w_{j}=\exp \left(-Q_{j}\right) \in \mathcal{F}_{\lambda}\left(C^{3}+\right)(0<\lambda<3 / 2)$, $j=1,2, \cdots, s$, and let (3.6) hold. Let $n \geq 1,1 \leq p \leq \infty$. Then we have

$$
\begin{aligned}
& \left\|W\left(f-v_{n}^{[s]}(f)\right)\right\|_{L^{p}\left(\mathbb{R}^{s}\right)} \\
& \leq \sum_{j=1}^{s} C_{j}\left\|W_{j}\left(\prod_{k=1}^{j-1} T_{k}^{1 / 4}\right) \bar{\omega}_{p, j}\left(f_{\hat{X}_{j}}, T_{j}^{1 / 4} w_{j} ; c_{j} \frac{a_{n}^{(j)}}{n}\right)\right\|_{L^{p}\left(\mathbb{R}_{j}^{s-1}\right)},
\end{aligned}
$$

where $\mathbb{R}_{j}^{s-1}$ is defined in (3.8). Now, we suppose that $f$ is continuous on $\mathbb{R}^{s}$. Let $\left\|W^{\delta} f\right\|_{L^{\infty}(\mathbb{R})}<\infty$ for some $0<\delta<1$, and let $W^{\delta} f \in \operatorname{Lip}(\alpha)$ for some $0<\alpha \leq 1$, that is,

$$
\left|W^{\delta} f\left(X_{1}\right)-W^{\delta} f\left(X_{2}\right)\right| \leq\left|X_{1}-X_{2}\right|^{\alpha} .
$$

Then we have

$$
\begin{aligned}
& \sum_{j=1}^{s} C_{j}\left\|W_{j}\left(\prod_{k=1}^{j-1} T_{k}^{1 / 4}\right) \bar{\omega}_{p, j}\left(f_{\hat{X}_{j}}, T_{j}^{1 / 4} w_{j} ; c_{j} \frac{a_{n}^{(j)}}{n}\right)\right\|_{L^{p}\left(\mathbb{R}_{j}^{s-1}\right)} \\
& \leq C \sum_{i=1}^{s}\left(\frac{a_{n}^{(i)}}{n}\right)^{\alpha} .
\end{aligned}
$$

(2) We suppose $w_{j}=\exp \left(-Q_{j}\right) \in \mathcal{F}\left(C^{2}+\right), \quad j=1,2, \cdots, s$, and hold (3.6). Let $n \geq 1,1 \leq p \leq \infty$. Then we have

$$
\left\|\frac{W\left(f-v_{n}^{[s]}(f)\right)}{\prod_{k=1}^{s} T_{k}^{1 / 4}}\right\|_{L^{p}\left(\mathbb{R}^{s}\right)} \leq \sum_{j=1}^{s} C_{j}\left\|W_{j} \bar{\omega}_{p, j}\left(f_{\hat{X}_{j}}, w_{j} ; c_{j} \frac{a_{n}^{(j)}}{n}\right)\right\|_{L^{p}\left(\mathbb{R}_{j}^{s-1}\right)} .
$$

Now, we suppose that $f$ has the conditions as (1). Then we have

$$
\sum_{j=1}^{s} C_{j}\left\|W_{j} \bar{\omega}_{p, j}\left(f_{\hat{X}_{j}}, w_{j} ; c_{j} \frac{a_{n}^{(j)}}{n}\right)\right\|_{L^{p}\left(\mathbb{R}_{j}^{s-1}\right)} \leq C \sum_{i=1}^{s}\left(\frac{a_{n}^{(i)}}{n}\right)^{\alpha} .
$$

Proof. We suppose $0<\delta<1$.

(1) (7.2) follows from (3.7). We will show (7.3). Now, we use $T^{\langle j-1\rangle}:=\prod_{i=1}^{j-1} T_{i}^{1 / 4}$, where $T^{\langle 0\rangle}=1$, and $W_{j}:=\prod_{1 \leq i \leq s, i \neq j} w_{i}$. We will estimate

$$
\left\|W_{j} T^{\langle j-1\rangle}\left(\frac{1}{t} \int_{0}^{t}\left\|T_{j}^{1 / 4}(x) w_{j}(x)\left(\Delta_{h \Phi_{t, j}(x)} f_{\hat{X}_{j}}(x)\right)\right\|_{L^{p}\left(|x| \leq \sigma_{j}(2 t)\right)}^{p} \mathrm{~d} h\right)^{1 / p}\right\|_{L^{p}\left(\mathbb{R}_{j}^{s-1}\right)} .
$$


From $W^{\delta} f \in \operatorname{Lip}(\alpha)$ and

$$
\begin{aligned}
& w_{j}(x) \sim w\left(x+(h / 2) \Phi_{t, j}(x)\right) \sim w\left(x-(h / 2) \Phi_{t, j}(x)\right) \quad \text { (see[3], Lemma 7) we have } \\
& \left(\frac{1}{t} \int_{0}^{t}\left\|T_{j}^{1 / 4}(x) w_{j}(x) \Delta_{h \Phi_{t, j}(x)} f_{\hat{X}_{j}}(x)\right\|_{L^{p}\left(|x| \leq \sigma_{j}(2 t)\right)}^{p} \mathrm{~d} h\right)^{1 / p} \\
& =\left(\frac{1}{t} \int_{0}^{t}\left\|T_{j}^{1 / 4}(x) w_{j}(x)\left\{f_{\hat{X}_{j}}\left(x+\frac{h}{2} \Phi_{t, j}(x)\right)-f_{\hat{X}_{j}}\left(x-\frac{h}{2} \Phi_{t, j}(x)\right)\right\}\right\|_{L^{p}\left(|x| \leqslant \sigma_{j}(2 t)\right)}^{p} \mathrm{~d} h\right)^{1 / p} \\
& \leq\left(\frac{1}{t} \int_{0}^{t} \| \frac{T_{j}^{1 / 4}(x) w_{j}(x)}{w_{j}^{\delta}\left(x+\frac{h}{2} \Phi_{t, j}(x)\right)}\left[\left|w_{j}^{\delta}\left(x+\frac{h}{2} \Phi_{t, j}(x)\right) f_{\hat{X}}\left(x+\frac{h}{2} \Phi_{t, j}(x)\right)-w_{j}^{\delta}\left(x-\frac{h}{2} \Phi_{t, j}(x)\right) f_{\hat{X}}\left(x-\frac{h}{2} \Phi_{t, j}(x)\right)\right|\right.\right. \\
& \left.\left.+\left|\left\{w_{j}^{\delta}\left(x+\frac{h}{2} \Phi_{t, j}(x)\right)-w_{j}^{\delta}\left(x-\frac{h}{2} \Phi_{t, j}(x)\right)\right\} f_{\hat{X}_{j}}\left(x-\frac{h}{2} \Phi_{t, j}(x)\right)\right|\right]\left.\right|_{L^{p}\left(|x| \leq \sigma_{j}(2 t)\right)} ^{p} \mathrm{~d} h\right)^{1 / p} \\
& \leq C\left(\frac { 1 } { t } \int _ { 0 } ^ { t } | | T _ { j } ^ { 1 / 4 } ( x ) w _ { j } ^ { ( 1 - \delta ) } ( x ) \left[\left|w_{j}^{\delta}\left(x+\frac{h}{2} \Phi_{t, j}(x)\right) f_{\hat{X}}\left(x+\frac{h}{2} \Phi_{t, j}(x)\right)-w_{j}^{\delta}\left(x-\frac{h}{2} \Phi_{t, j}(x)\right) f_{\hat{X}}\left(x-\frac{h}{2} \Phi_{t, j}(x)\right)\right|\right.\right. \\
& \left.\left.+\left|\left\{w_{j}^{\delta}\left(x+\frac{h}{2} \Phi_{t, j}(x)\right)-w_{j}^{\delta}\left(x-\frac{h}{2} \Phi_{t, j}(x)\right)\right\} f_{\hat{X}_{j}}\left(x-\frac{h}{2} \Phi_{t, j}(x)\right)\right|\right]\left.\right|_{L^{p}\left(|x| \leq \sigma_{j}(2 t)\right)} ^{p} \mathrm{~d} h\right)^{1 / p} \\
& =: I_{1}+I_{2} \text {. }
\end{aligned}
$$

By (7.2) we see

$$
\begin{aligned}
& \left\|W_{j} T^{\langle j-1\rangle} I_{1}\right\|_{L^{p}\left(\mathbb{R}^{s-1}\right)}=\| W_{j}^{1-\delta} T^{\langle j-1\rangle}\left(\frac{1}{t} \int_{0}^{t} \|\left(W_{\hat{X}_{j}}^{\delta} f_{\hat{X}_{j}}\right)\left(x+\frac{h}{2} \Phi_{t, j}(x)\right)\right. \\
& \left.-\left(W_{\hat{X}_{j}}^{\delta} f_{\hat{X}_{j}}\right)\left(x-\frac{h}{2} \Phi_{t, j}(x)\right) \|_{L^{p}\left(|x| \leq \sigma_{j}(2 t)\right)}^{p} \mathrm{~d} h\right)^{1 / p} \|_{L^{p}\left(\mathbb{R}^{s-1}\right)} \\
& \leq C\left\|W_{j}^{1-\delta} T^{\langle j-1\rangle}\left(\frac{1}{t} \int_{0}^{t} h^{p \alpha} \mathrm{d} h\right)^{1 / p}\right\|_{L^{p}\left(\mathbb{R}^{s-1}\right)} \\
& \leq t^{\alpha}\left\|W_{j}^{1-\delta} T^{\langle j-1\rangle}\right\|_{L^{p}\left(\mathbb{R}^{s-1}\right)} \leq C t^{\alpha} .
\end{aligned}
$$

On the other hand, for $I_{2}$ we estimate

$$
\begin{aligned}
& =C \| W_{j} T^{\langle j-1\rangle}\left(\frac{1}{t} \int_{0}^{t} \| T_{j}^{1 / 4}(x) w_{j}^{(1-\delta) / 2}(x) h \Phi_{t, j}(x) w_{j}^{\delta}(\xi)\right. \\
& \left.\times \delta Q_{j}^{\prime}(\xi) f_{\hat{x}_{j}}\left(x-\frac{h}{2} \Phi_{t, j}(x)\right) \|_{L^{p}\left(|x| \leqslant \sigma_{j}(2 t)\right)}^{p} \mathrm{~d} h\right)^{1 / p} \|_{L^{p}\left(\mathbb{R}^{s-1}\right)}, \\
& x-\frac{h}{2} \Phi_{t, j}(x)<\xi<x+\frac{h}{2} \Phi_{t, j}(x), \\
& \leq C\left\|W_{j}^{1-\delta} T^{\langle j-1\rangle}\left(\frac{1}{t} \int_{0}^{t} h^{p}\left|w_{j}^{(1-\delta) / 2}(x) Q_{j}^{\prime}(\xi)\left(W_{\hat{X}_{j}}^{\delta} f_{\hat{X}_{j}}\right)\left(x-\frac{h}{2} \Phi_{t, j}(x)\right)\right| \mathrm{d} h\right)^{1 / p}\right\|_{L^{p}\left(\mathbb{R}^{s}\right)},
\end{aligned}
$$


by the boundedness of $T_{j}^{1 / 4}(x) w_{j}^{(1-\delta) / 2}(x)$

$$
\leq C t\left\|W^{\delta} f\right\|_{L^{p}\left(\mathbb{R}^{s}\right)}
$$

by the boundedness of $w_{j}^{(1-\delta) / 2}(x)\left|Q_{j}^{\prime}(\xi)\right|$ (note $\left|Q^{\prime}\right| \leq Q^{C}$ ) and Lemma 3.10

$$
\leq C t^{\alpha}\left(\text { by } w^{\delta} f \in L^{p}(\mathbb{R})\right) \text {. }
$$

Hence we conclude

$$
\left\|W_{j} T^{\langle j-1\rangle}\left(\frac{1}{t} \int_{0}^{t}\left\|T_{j}^{1 / 4}(x) w_{j}(x)\left(\Delta_{h \Phi_{t, j}(x)} f_{\hat{x}_{j}}(x)\right)\right\|_{L^{p}\left(|x| \leq \sigma_{j}(2 t)\right)}^{p} \mathrm{~d} h\right)^{1 / p}\right\|_{L^{p}\left(\mathbb{R}_{j}^{s-1}\right)} \leq C t^{\alpha} .
$$

Now, we see

$$
\begin{aligned}
& \left\|W_{j} T^{\langle j-1\rangle} \inf _{c_{j} \text { constant) }}\right\|\left(f_{\hat{X}_{j}}(x)-c_{j}\right) w_{j}(x)\left\|_{L^{p}\left(\mathbb{R} \backslash\left[-\sigma_{j}(4 t), \sigma_{j}(4 t)\right]\right)}\right\|_{L^{p}\left(\mathbb{R}_{j}^{s-1}\right)} \\
& \leq\left\|W_{j} T^{\langle j-1\rangle}\right\| f_{\hat{X}_{j}}(x) w_{j}(x)\left\|_{L^{p}\left(\mathbb{R} \backslash\left[-\sigma_{j}(4 t), \sigma_{j}(4 t)\right]\right)}\right\|_{L^{p}\left(\mathbb{R}_{j}^{s-1}\right)} \\
& \leq w_{j}^{1-\delta}\left(\sigma_{j}(4 t)\right)\left\|W^{\delta} f\right\|_{L^{p}\left(\mathbb{R}^{s}\right)} \leq C w_{j}^{1-\delta}\left(\sigma_{j}(4 t)\right) .
\end{aligned}
$$

Here, if we set $4 t=a_{u} / u$, then we see

$$
w_{j}^{1-\delta}\left(\sigma_{j}(4 t)\right)=\exp \left(-(1-\delta) Q_{j}\left(a_{u}\right)\right) \sim \exp \left(-C \frac{u}{\sqrt{T\left(a_{u}\right)}}\right) \leq \mathrm{e}^{-u^{\eta}}
$$

for some $0<\eta<1$, that is,

$$
w_{j}^{1-\delta}\left(\sigma_{j}(4 t)\right) \leq C \mathrm{e}^{-u^{\delta}} \leq C \frac{a_{u}}{4 u}=C t .
$$

Therefore, we have

$$
\left\|W_{j} T^{\langle j-1\rangle} \inf _{\left.c_{j} \text { (constant) }\right)}\right\|\left(f_{\hat{x}_{j}}(x)-c_{j}\right) w_{j}(x)\left\|_{L^{p}\left(\mathbb{R}\left[-\sigma_{j}(4 t), \sigma_{j}(4 t)\right]\right)}\right\| \|_{L^{p}\left(\mathbb{R}_{j}^{s-1}\right)} \leq C t .
$$

Consequently, by (7.6) and (7.7) we have

$$
\left\|W_{j} T^{\langle j-1\rangle} \bar{\omega}_{p, j}\left(f_{\hat{X}_{j}}, w_{j} ; t\right)\right\|_{L^{p}\left(\mathbb{R}_{j}^{s-1}\right)} \leq C t^{\alpha} .
$$

So we have (7.3), that is,

$$
\sum_{j=1}^{s} C_{j}\left\|W_{j} T^{\langle j-1\rangle} \bar{\omega}_{p, j}\left(f_{\hat{X}_{j}}, w_{j} ; c_{j} \frac{a_{n}^{(j)}}{n}\right)\right\|_{L^{p}\left(\mathbb{R}_{j}^{s-1}\right)} \leq C \sum_{i=1}^{s}\left(\frac{a_{n}^{(i)}}{n}\right)^{\alpha} .
$$

(2) (7.4) follows from (3.7). The estimate (7.5) follows as (1). We omit the proof. \# 


\section{Approximation for Differentiable Functions}

In this section, we treat the differentiable functions.

Let $s>1, r \geq 1$ be fixed integers, and let $w_{j} \in \mathcal{F}_{\lambda}\left(C^{3}+\right), j=1, \cdots, s$. We suppose that the multivariate function $f\left(x_{1}, \cdots, x_{s}\right)$ is $r$-times partial differentiable, and then with norm:

$$
\|W f\|_{\mathcal{W}_{r, p}}:=\sum_{i=1}^{s}\left\|w_{i} T_{i}^{1 / 4} D_{i}^{r} f\right\|_{L^{p}\left(\mathbb{R}^{s}\right)}<\infty,
$$

where

$$
D_{i}^{r} f:=\frac{\partial^{r} f}{\partial^{r} x_{i}}, i=1,2, \cdots, s .
$$

The class of all functions $f\left(x_{1}, \cdots, x_{s}\right)$ with $\|W f\|_{\mathcal{W}_{r, p}}<\infty$ will be denoted by $\mathcal{W}_{r, p}$. In the sequel, if $1 \leq i \leq s$ is an integer, then $\|f\|_{L_{p, i}(\mathbb{R})}$ will denote the $L_{p}$-norm of $f$ taken with respect to the $i$-th variable.

Theorem 8.1. We suppose $w_{j}=\exp \left(-Q_{j}\right) \in \mathcal{F}_{\lambda}\left(C^{3}+\right)$ and $r \geq 1$ is an integer. Let $n \geq 1,1 \leq p \leq \infty$, and let $W f \in \mathcal{W}_{r, p}$. Then we have

$$
\left\|W\left(f-v_{n}^{[s]}(f)\right)\right\|_{L^{p}\left(\mathbb{R}^{s}\right)} \leq C\left(\frac{a_{n}}{n}\right)^{r}\|W f\|_{\mathcal{W}_{r, p}},
$$

where $a_{n}=\max \left\{a_{n}^{(i)}, i=1,2, \cdots, s\right\}$.

Remark 8.2. Especially, for $r=1$ we have

$$
\left\|W\left(f-v_{n}^{[s]}(f)\right)\right\|_{L^{p}\left(\mathbb{R}^{s}\right)} \leq C\left(\frac{a_{n}}{n}\right) \sum_{i=1}^{s}\left\|w_{i} T_{i}^{1 / 4} D_{i}^{1} f\right\|_{L^{p}\left(\mathbb{R}^{s}\right)} .
$$

Theorem 8.3 ([7], Cororally 8 ). We suppose $w \in \mathcal{F}\left(C^{2}+\right)$. Let $1 \leq p \leq \infty$ and $r(\geq 1)$ is an integer. If $g^{(r-1)}$ be absolutely continuous and $w g^{(r)} \in L^{p}(\mathbb{R})$, then we have

$$
E_{p, n}(g) \leq C\left(\frac{a_{n}}{n}\right)^{r}\left\|w g^{(r)}\right\|_{L^{p}(\mathbb{R})} .
$$

Equivalently,

$$
E_{p, n}(g) \leq C\left(\frac{a_{n}}{n}\right)^{r} E_{p, n-1}\left(g^{(r)}\right)
$$

Proof of Theorem 8.1. We use Proposition 2.3, that is, $T^{\alpha} w \sim w_{\alpha} \in \mathcal{F}\left(C^{2}+\right)$. In view of Theorem 3.3 (1) and a repeated application of Theorem 8.3, we get

$$
\begin{aligned}
& \left\|w_{1}(x)\left(f_{\hat{X}_{1}}-v_{n}^{[s]}\left(f_{\hat{X}_{1}}\right)\right)\right\|_{L^{p}(\mathbb{R})} \\
& \leq E_{p, n}\left(T_{1}^{1 / 4} w_{1}, f_{\hat{X}_{1}}\right) \leq C\left(\frac{a_{n}^{(1)}}{n}\right)^{r}\left\|T_{1}^{1 / 4}(x) w_{1}(x) f_{\hat{X}_{1}}^{(r)}\right\|_{L^{p}(\mathbb{R})} \\
& =C_{1}\left(\frac{a_{n}^{(1)}}{n}\right)^{r}\left\|w_{1}(x) T_{1}^{1 / 4}(x) D_{1}^{r} f\right\|_{L^{p}(\mathbb{R})},
\end{aligned}
$$


where the constant $C_{1}$ is independent of $\hat{X}_{1}$, and $D_{1}$ denotes differentiation with respect to the first variable. Similarly, for $j=1,2, \cdots, s$,

$$
\left\|w_{j}\left(f_{\hat{X}_{j}}-v_{n, j}\left(f_{\hat{X}_{j}}\right)\right)\right\|_{L^{p}(\mathbb{R})} \leq C_{j}\left(\frac{a_{n}^{(j)}}{n}\right)^{r}\left\|w_{j} T_{j}^{1 / 4} D_{j}^{r} f\right\|_{L^{p}(\mathbb{R})},
$$

where $D_{j}^{r}$ denotes the derivative with respect to the $j$-th variable.

Using Lemma 3.7 and (8.2), we obtain for integer $j \geq 2$,

$$
\begin{aligned}
& \left\|W\left(v_{n}^{[j-1]}(f)-v_{n}^{[j]}(f)\right)\right\|_{\left.L^{p} \mathbb{R}^{s}\right)}=\left\|W v_{n}^{[j-1]}\left(f-v_{n, j}(f)\right)\right\|_{L^{p}\left(\mathbb{R}^{s}\right)} \\
& \leq C\left\|W\left(\prod_{i=1}^{j-1} T_{i}^{1 / 4}\right)\left(f-v_{n, j}(f)\right)\right\|_{L^{p}\left(\mathbb{R}^{s}\right)} \leq\left(\frac{a_{n}^{(j)}}{n}\right)^{r}\left\|w_{j} T_{j}^{1 / 4} D_{j}^{r} f\right\|_{L^{p}\left(\mathbb{R}^{s}\right)} .
\end{aligned}
$$

From (8.2) and (8.3), we get

$$
\begin{aligned}
& \left\|W\left(f-v_{n}^{[s]}(f)\right)\right\|_{L^{p}\left(\mathbb{R}^{s}\right)} \\
& =\left\|W\left(f-v_{n}^{[1]}(f)\right)\right\|_{L^{p}\left(\mathbb{R}^{s}\right)}+\sum_{j=2}^{s}\left\|W\left(v_{n}^{[j-1]}(f)-v_{n}^{[j]}(f)\right)\right\|_{L^{p}\left(\mathbb{R}^{s}\right)} \\
& \leq C\left(\frac{a_{n}}{n}\right)^{r} \sum_{j=1}^{s}\left\|W\left(\prod_{i=1}^{j} T_{i}^{1 / 4}\left(x_{i}\right)\right) D_{j}^{r} f\right\|_{L^{p}\left(\mathbb{R}^{s}\right)} .
\end{aligned}
$$

Therefore, we conclude

$$
\left\|W\left(f-v_{n}^{[s]}(f)\right)\right\|_{L^{p}\left(\mathbb{R}^{s}\right)} \leq C\left(\frac{a_{n}}{n}\right)^{r} \sum_{j=1}^{s}\left\|W T^{\langle s\rangle} D_{j}^{(r)} f\right\|_{L^{p}\left(\mathbb{R}^{s}\right)}=C\left(\frac{a_{n}}{n}\right)^{r}\|W f\|_{W_{r, p}} \quad \quad \#
$$

\section{References}

[1] Jung, H.S. and Sakai, R. (2009) Specific Examples of Exponential Weights. Communications of the Korean Mathematical Society, 24, 303-319.

https://doi.org/10.4134/CKMS.2009.24.2.303

[2] Levin, A.L. and Lubinsky, D.S. (2001) Orthogonal Polynomials for Exponential Weights. Springer, New York. https://doi.org/10.1007/978-1-4613-0201-8

[3] Sakai, R. and Suzuki, N. (2013) Mollification of Exponential Weights and Its Application to the Markov-Bernstein Inequality. The Pioneer Journal of Mathematics, 7, 83-101.

[4] Rudin, W. (1987) Real and Complex Analysis. 3rd Edition, McGraw-Hill, New York.

[5] Itoh, K., Sakai, R. and Suzuki, N. (2015) The de la Vallée Poussin Mean and Polynomial Approximation for Exponential Weights. International Journal of Analysis, 2015, Article ID: 706930.

[6] Damelin, S.B. and Lubinsky, D.S. (1998) Jackson Theorems for Erdös Weights in $L_{p}(0<p \leq \infty)$. Journal of Approximation Theory, 94, 333-382.

[7] Sakai, R. and Suzuki, N. (2011) Favard-Type Inequalities for Exponential Weights. The Pioneer Journal of Mathematics, 3, 1-16.

[8] Sakai, R. (2017) A Study of Weighted Polynomial Approximations for Orthogonal 
Polynomial Expansion. Journal of Advances in Applied Mathematics, 173-195.

[9] Mhaskar, H.N. (1996) Introduction to the Theory of Weighted Polynomial Approximation. World Scientific, Singapore.

[10] Itoh, K., Sakai, R. and Suzuki, N. (2015) An estimate for Derivative of the de la Vallée Poussin Mean. Mathematical Journal of Ibaraki University, 47, 1-18. https://doi.org/10.5036/mjiu.47.1

[11] Damelin, S.B. (1998) Converse and Smoothness Theorems for Erdös Weights in $L_{p}(0<p \leq \infty)$. Journal of Approximation Theory, 93, 349-398.

[12] Itoh, K., Sakai, R. and Suzuki, N. Uniform Convergence of Orthogonal Polynomial Expansions for Exponential Weights. (Preprint) 


\section{Appendix}

In this Appendix we state two inequalities which play important roles in the study of approximation theory. In fact, we use Theorem $A_{1}$ in the proof of Theorem 5.2. Let $a_{n}^{(i)}$ be the MRS number of $w_{i}=\exp \left(-Q_{i}\right)$.

Theorem $A_{1}$ (Markov-Bernstein inequalities). Let $0<p \leq \infty$, and let $r_{1}, \cdots, r_{s} \geq 0$ be integers. There exists $C \neq C(n, P)>0$ such that for $n \geq 1$ and $P \in \mathcal{P}_{n ; s}\left(\mathbb{R}^{s}\right)$.

(1) if $w_{i} \in \mathcal{F}\left(C^{2}+\right)$, then we have

$$
\left\|P(X)^{\left(r_{1}, \cdots, r_{j}\right)} W(X)\right\|_{L^{p}\left(\mathbb{R}^{s}\right)} \leq C \prod_{i=1}^{s}\left(\frac{n\left(T_{i}\left(a_{n}^{(i)}\right)\right)^{1 / 2}}{a_{n}^{(i)}}\right)^{r_{i}}\|P(X) W(X)\|_{L^{p}\left(\mathbb{R}^{s}\right)} .
$$

(2) if $w_{i} \in \mathcal{F}_{\lambda}\left(C^{3}+\right)$, then we have

$$
\left\|\frac{P(X)^{\left(r_{1}, \cdots, r_{j}\right)} W(X)}{\prod_{i=1}^{s} T_{i}^{r_{i} / 2}}\right\|_{L^{p}\left(\mathbb{R}^{s}\right)} \leq C \prod_{i=1}^{s}\left(\frac{n}{a_{n}^{(i)}}\right)^{r_{i}}\|P(X) W(X)\|_{L^{p}\left(\mathbb{R}^{s}\right)} .
$$

The following, so called, Nikolskee-type inequality is useful.

Theorem $A_{2}$ (Nikolskii-type inequality). Let

$w_{i}=\exp (-Q) \in \mathcal{F}_{\lambda}\left(C^{3}+\right)(0<\lambda<3 / 2)$, and let $P \in \mathcal{P}_{n ; s}$. For $0<p \leq q \leq \infty$, we have

$$
\|W P\|_{L_{p}\left(\mathbb{R}^{s}\right)} \leq C \prod_{i=1}^{s}\left(a_{n}^{(i)}\right)^{\frac{1}{p}-\frac{1}{q}}\|W P\|_{L_{q}\left(\mathbb{R}^{s}\right)},
$$

and for $1 \leq q<p \leq \infty$, we have

$$
\left\|\prod_{i=1}^{s} T_{i}^{\frac{1}{2}\left(\frac{1}{p}-\frac{1}{q}\right)} W P\right\|_{L_{p}\left(\mathbb{R}^{s}\right)} \leq C \prod_{i=1}^{s}\left(\frac{n}{a_{n}^{(i)}}\right)^{\frac{1}{q}-\frac{1}{p}}\|W P\|_{L_{q}(\mathbb{R})} .
$$

To prove Theorem $A_{1}$ we need the Proposition 5.3.

Proof of Theorem $A_{1}$. To prove (1), we use the second inequality in Proposition 5.3, repeatedly. Then we easily obtain the result.

(2) Using the first inequality in Proposition 5.3, repeatedly, we have the result. \#

The proof of Theorem $A_{2}$ is obtained by repeatedly using the following proposition.

Proposition $A_{3}$ ([8], Theorem 18). Let $w=\exp (-Q) \in \mathcal{F}_{\lambda}\left(C^{3}+\right)(0<\lambda<3 / 2)$, and let $P \in \mathcal{P}_{n}$. For $0<p \leq q \leq \infty$, we have

$$
\|w P\|_{L_{p}(\mathbb{R})} \leq C a_{n}^{\frac{1}{p}-\frac{1}{q}}\|w P\|_{L_{q}(\mathbb{R})},
$$

and for $1 \leq q<p \leq \infty$, we have

$$
\left\|T^{\frac{1}{2}\left(\frac{1}{p}-\frac{1}{q}\right)} w P\right\|_{L_{p}(\mathbb{R})} \leq C\left(\frac{n}{a_{n}}\right)^{\frac{1}{q}-\frac{1}{p}}\|w P\|_{L_{q}(\mathbb{R})} .
$$


Submit or recommend next manuscript to SCIRP and we will provide best service for you:

Accepting pre-submission inquiries through Email, Facebook, LinkedIn, Twitter, etc. A wide selection of journals (inclusive of 9 subjects, more than 200 journals)

Providing 24-hour high-quality service

User-friendly online submission system

Fair and swift peer-review system

Efficient typesetting and proofreading procedure

Display of the result of downloads and visits, as well as the number of cited articles Maximum dissemination of your research work

Submit your manuscript at: http://papersubmission.scirp.org/

Or contact am@scirp.org 